\title{
MERGING BLACK HOLE BINARIES IN GALACTIC NUCLEI: IMPLICATIONS FOR ADVANCED-LIGO DETECTIONS
}

\author{
Fabio Antonini and Frederic A. Rasio \\ Center for Interdisciplinary Exploration and Research in Astrophysics (CIERA) and Department of Physics and Astrophysics, \\ Northwestern University, Evanston, IL 60208, USA \\ Received 2016 June 16; revised 2016 August 18; accepted 2016 August 18; published 2016 November 7
}

\begin{abstract}
Motivated by the recent detection of gravitational waves from the black hole binary merger GW150914, we study the dynamical evolution of (stellar-mass) black holes in galactic nuclei, where massive star clusters reside. With masses of $\sim 10^{7} M_{\odot}$ and sizes of only a few parsecs, nuclear star clusters (NSCs) are the densest stellar systems observed in the local universe and represent a robust environment where black hole binaries can dynamically form, harden, and merge. We show that due to their large escape speeds, NSCs can retain a large fraction of their merger remnants. Successive mergers can then lead to significant growth and produce black hole mergers of several tens of solar masses similar to GW150914 and up to a few hundreds of solar masses, without the need to invoke extremely low metallicity environments. We use a semi-analytical approach to describe the dynamics of black holes in massive star clusters. Our models give a black hole binary merger rate of $\approx 1.5 \mathrm{Gpc}^{-3} \mathrm{yr}^{-1}$ from NSCs, implying up to a few tens of possible detections per year with Advanced LIGO. Moreover, we find a local merger rate of $\sim 1 \mathrm{Gpc}^{-3} \mathrm{yr}^{-1}$ for high mass black hole binaries similar to GW150914; a merger rate comparable to or higher than that of similar binaries assembled dynamically in globular clusters (GCs). Finally, we show that if all black holes receive high natal kicks, $\gtrsim 50 \mathrm{~km} \mathrm{~s}^{-1}$, then NSCs will dominate the local merger rate of binary black holes compared to either GCs or isolated binary evolution.
\end{abstract}

Key words: galaxies: nuclei - gravitational waves - stars: black holes

\section{INTRODUCTION}

On 2015 September 14, the Advanced LIGO interferometer (aLIGO) detected the event GW150914, which has been interpreted as the first direct observation of gravitational waves (GWs) from the inspiral and merger of a pair of black holes (BHs; Abbott et al. 2016a). The event GW150914 was produced by two BHs with masses of $36_{-4}^{+5} M_{\odot}$ and $29_{-4}^{+4} M_{\odot}$ (in the source frame), at a redshift $z \approx 0.1$ assuming standard cosmology (Abbott et al. 2016b). The detection of the GW signal of GW150914 has provided the first direct evidence that black holes with masses $\gtrsim 30 M_{\odot}$ exist and that they can reside in binary systems. Assuming that the source-frame binary $\mathrm{BH}$ merger rate is constant within the volume in which GW150914 could have been detected, and that GW150914 is representative of the underlying binary $\mathrm{BH}$ population, the $\mathrm{BH}-\mathrm{BH}$ merger rate is inferred to be $2-53 \mathrm{Gpc}^{-3} \mathrm{yr}^{-1}$ in the comoving frame (Abbott et al. 2016c).

Abbott et al. (2016d) reviews various channels for the formation of BH binaries that can coalesce within a Hubble time thus becoming potentially detectable by aLIGO. These include the dynamical formation in dense stellar environments (e.g., Portegies Zwart \& McMillan 2000; Miller \& Lauburg 2009; Banerjee et al. 2010; Downing et al. 2011; Rodriguez et al. 2015), and isolated binary evolution (e.g., Belczynski et al. 2002, 2016; Dominik et al. 2012; Spera et al. 2015; de Mink \& Mandel 2016). While most of the former literature focused on $\mathrm{BH}$ binaries forming in globular clusters (GCs), little attention has been devoted to the formation of such binaries in nuclear star clusters (NSCs). However, NSCs have total stellar masses that are comparable to the whole stellar mass of the GC system for the galaxy, at least in the Milky Way and are the densest and most massive star clusters observed in the local universe (e.g., Böker et al. 2004; Côté et al. 2006), therefore, representing a natural environment where dynamical processes can efficiently lead to the formation of $\mathrm{BH}$ binaries. In this paper, we consider the dynamical formation scenario and explore the contribution to the BH-binary merger rate from NSCs.

In a stellar cluster, stellar-mass BHs formed from the death of massive stars, quickly segregate to the center through dynamical friction (Chandrasekhar 1943; Spitzer 1987). In these high density environments, BHs can efficiently interact with each other and dynamically form new binaries. Such binaries will subsequently harden through three-body interactions (Heggie 1975). Via such dynamical processes, GCs can produce a significant population of $\mathrm{BH}$ binaries that, after being ejected from the cluster, will be able to merge in the local universe (e.g., Rodriguez et al. 2016a). Over the last years, our understanding of the evolution of BHs in star clusters has improved considerably thanks to numerical efforts (e.g., Aarseth 2012; Morscher et al. 2013, 2015; Wang et al. 2015). However, the role of NSCs and their contribution to the BH-binary merger rate in the local universe remains quite obscure. As discussed in what follows, NSCs differ from lower mass GCs in at least three important ways, each of these can significantly enhance the $\mathrm{BH}$ merger rate and affect the properties of the merging binaries in NSCs.

(1) NSCs retain most of their BHs. While natal kicks can easily eject BHs from GCs, the natal kick magnitudes are unlikely to be large enough to eject a considerable number of $\mathrm{BHs}$ from NSCs given the large escape speed in these latter systems. Whether dynamically formed BH binaries will merge, and whether the merger will occur inside the cluster, depends on the cluster escape speed. The low escape speed $\left(\lesssim 10 \mathrm{~km} \mathrm{~s}^{-1}\right)$ from low mass clusters $\left(M_{\mathrm{cl}} \lesssim 10^{5} M_{\odot}\right)$, implies that most BH binaries are ejected early after their formation with an orbital semimajor axis, which is typically too large for GW emission to become efficient and drive the merger of the 
binary in one Hubble time. The vast majority of dynamically formed $\mathrm{BH}$ binaries in GCs are also kicked out before merging, but they are able to merge in the local universe (e.g., Rodriguez et al. 2015). As argued by Miller \& Lauburg (2009), given that NSCs have escape speeds that are several times those of globulars, they can retain most of their $\mathrm{BH}$ binaries. Moreover, as we show below, even when accounting for the recoil kick due to anisotropic emission of GW radiation, a large fraction of merger products is likely to be retained inside NSCs.

(2) NSCs contain young stellar populations. The common finding emerging from spectroscopic surveys is that NSCs are characterized by complex star formation histories with a mixture of morphological components and different stellar populations spanning a wide range of characteristic ages (from $10 \mathrm{Myr}$ to $10 \mathrm{Gyr}$ ) and metallicities (Figer et al. 2004; Rossa et al. 2006; Walcher et al. 2006; Do et al. 2015). This implies that unlike GCs, NSCs can still form fresh BHs and $\mathrm{BH}$ binaries at the present time. The presence of significant additional gas not found in old GCs could also result in differences in the black hole mass distribution, as well as the dynamics of the underlying black hole population (Leigh et al. 2013, 2014).

(3) NSCs reside at the center of galaxies. Therefore, unlike GCs, NSCs are not isolated. In time, newly formed star clusters could migrate by dynamical friction from the galaxy into the NSC itself replenishing BHs that have been kicked out by three-body processes or by GW recoil kicks. The orbital decay of massive star clusters through dynamical friction constitutes an additional source, which can repopulate the BH-binary population in the nuclei of galaxies (Antonini 2014).

In this paper, we study the dynamical formation of $\mathrm{BH}$ binary mergers in NSCs, with particular focus on NSCs that do not host a central massive black hole $(\mathrm{MBH})$, which we define here as BHs having masses of $\gtrsim 10^{6} M_{\odot}$. Our cluster models are based on a semi-analytical approach, which describes the formation and evolution of $\mathrm{BH}$ binaries in static cluster models. Although necessarily approximated, these models are shown to give reasonable results when compared to recent Monte Carlo models of massive GCs (Chatterjee et al. 2016; Rodriguez et al. 2016a) and previous BH-binary merger rate estimates from NSCs (Portegies Zwart \& McMillan 2000; Miller \& Lauburg 2009). We stress that, although the MBH occupation fraction in NSCs is largely unconstrained observationally, it has been long recognized that some NSCs do not have MBHs (e.g., Merritt et al. 2001; Neumayer \& Walcher 2012). We note that NSCs with MBHs are very different, dynamically, than NSCs without. If an $\mathrm{MBH}$ is present, the velocity dispersion keeps growing toward the $\mathrm{MBH}$, which means that no binary will be hard all the way to the center. Here we make use of the semianalytical galaxy formation models presented in Antonini et al. (2015b) to predict the occupation fraction of MBHs in NSCs and the NSC initial mass function, which combined with the results of our cluster models allows an estimate of the aLIGO detection rate and properties of $\mathrm{BH}$ mergers forming in NSCs.

Our results suggest that the $\mathrm{BH}$ merger event rate from NSCs is substantial, with several tens of events per year detectable with aLIGO. In addition, we propose a new dynamical pathway to the formation of high mass BH-binary mergers similar to GW150914. This merger path is exclusive to NSCs and to the most massive GCs. Due to their large escape speeds, such massive clusters can keep a large fraction of their BH merger remnants while also evolving rapidly enough that the holes can

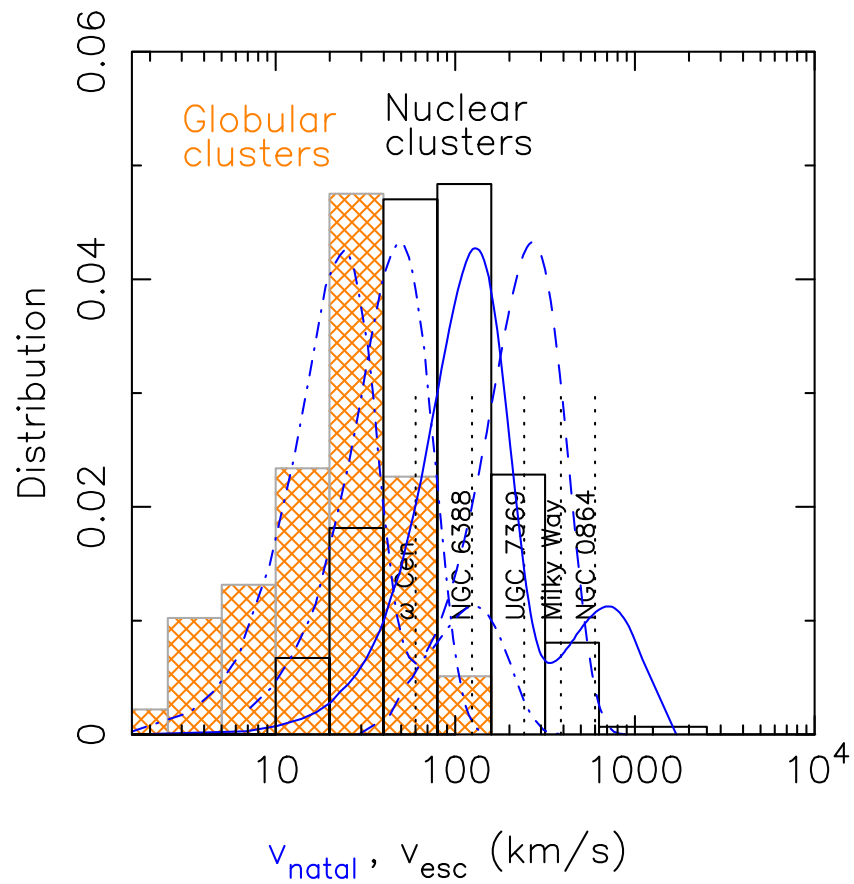

Figure 1. Distribution of escape velocities from NSCs and GCs (histograms) compared to distributions of natal kicks taken from Figure 3 of Repetto et al. (2012; blue curves). Blue solid and dashed lines correspond to distributions that are typically used to model the kick velocities of neutron stars. The solid line is the Arzoumanian distribution (Arzoumanian et al. 2002), the dashed line is the Hansen \& Phinney (1997) distribution. The two dotted-dashed lines are these two distributions but with kick speeds reduced, assuming that the momentum imparted to the black hole is the same as the momentum imparted to the neutron star. Note that if BHs receive natal kicks as large as those of neutron stars, most of them will be ejected from GCs but not from NSCs.

sink back to the central regions where they can form a new binary, which will subsequently harden and merge. We find that this process can repeat several times and produce $\mathrm{BH}$ mergers of several tens of solar masses and up to a few hundreds of solar masses, without the need of invoking extremely low metallicity environments.

The paper is organized as follows. In Sections 2, we discuss the processes leading to the formation and merger of $\mathrm{BH}$ binaries in the high density cores of GCs and NSCs, focusing on the processes that can lead to the full ejection of BHs. In Section 3, we describe our semi-analytical approach and derive the expected merger rate of $\mathrm{BH}$ binaries in NSCs. In Section 4, we discuss the implications of our results including the aLIGO detection rate and the contribution to the $\mathrm{BH}$ merger rate from NSCs hosting central MBHs. Finally, we summarize the main results of our study in Section 5.

\section{DYNAMICAL EVOLUTION OF BH BINARIES IN STELLAR CLUSTERS}

\subsection{Natal Kicks}

Due to asymmetries in the mass ejection or in the neutrino flux during core-collapse supernovae the black holes might receive appreciable natal kicks, which could eject them from the cluster. Thus, before we discuss the dynamical processes that can lead to the formation and merger of $\mathrm{BH}$ binaries in star clusters, it is useful to consider natal kicks as a phenomenon that can fully eject BHs from a star cluster, thereby aborting the dynamical formation channel for $\mathrm{BH}$ mergers. 
The histograms in Figure 1 show the distribution of escape velocities from NSCs and GCs. The escape velocities of GCs are central escape velocities calculated using the photometric data from the catalog by Harris (1996) and using single-mass King models with a constant mass-to-light ratio $M / L_{v}=3$. The NSCs escape velocities (from the cluster half-mass radius) were computed from the expression (e.g., Georgiev et al. 2009):

$$
v_{\mathrm{esc}} \approx f_{\mathrm{c}} \sqrt{\frac{M_{\mathrm{cl}}}{M_{\odot}} \frac{\mathrm{pc}}{r_{\mathrm{h}}}} \mathrm{km} \mathrm{s}^{-1}
$$

where $r_{\mathrm{h}}$ and $M_{\mathrm{cl}}$ are the cluster half-light radius and mass; the coefficient $f_{\mathrm{c}}$ takes into account the dependence of the escape velocity on the concentration of the cluster (i.e., $c=\log \left(r_{\mathrm{t}} / r_{\mathrm{c}}\right)$, with $r_{\mathrm{t}}$ and $r_{\mathrm{c}}$ the cluster tidal and core radii). The cluster radii and masses were taken from the sample of late-type galaxies of Georgiev et al. (2016). For more than half of the NSCs in these galaxies, Georgiev \& Böker (2014) find that a King profile with a high concentration index, $c=2$, provides the best fit. This concentration parameter corresponds to $f_{\mathrm{c}} \approx 0.1$ (King 1962) - this latter is the value of $f_{c}$ that we adopted in evaluating Equation (1). Figure 1 shows that escape velocities from NSCs are substantially larger than those from GCs, though the two distributions somewhat overlap near $M_{\mathrm{cl}} \sim 10^{6} M_{\odot}$, where the two type of systems have similar structural properties (e.g., Böker et al. 2004; Côté et al. 2006).

The distributions of natal kicks ( $v_{\text {natal }}$; blue lines) in Figure 1 were taken from Figure 3 of Repetto et al. (2012). These authors consider two different neutron star natal kick distributions. One is the Hansen \& Phinney (1997) distribution, the other is the bimodal distribution for neutron star kicks proposed by Arzoumanian et al. (2002) which has a lower peak at $\approx 1000 \mathrm{~km} \mathrm{~s}^{-1}$ and the higher peak at $\approx 100 \mathrm{~km} \mathrm{~s}^{-1}$. We also show two modified versions of these distributions (blue dotteddashed lines), which were obtained by assuming that the momentum imparted on a $\mathrm{BH}$ is the same as the momentum given to a neutron star taken from the two former distributions. Thus the kick velocities are reduced in these latter models by the neutron star to $\mathrm{BH}$ mass ratio (Repetto et al. 2012).

Figure 1 shows that $\mathrm{BHs}$ receiving natal kicks as large as $v_{\text {natal }} \gtrsim 50 \mathrm{~km} \mathrm{~s}^{-1}$ will escape from GCs before they can dynamically interact, which will suppress the dynamical formation of $\mathrm{BH}$ mergers in these systems. However, from Figure 1, we also see that BHs will be easily retained in NSCs even for natal kicks as large as a few $100 \mathrm{~km} \mathrm{~s}^{-1}$. Hence, if BHs receive natal kicks of $\gtrsim 50 \mathrm{~km} \mathrm{~s}^{-1}$, we expect that this will greatly reduce the $\mathrm{BH}$ merger rate from GCs (e.g., Chatterjee et al. 2016) as well as that from isolated binary evolution (e.g., Belczynski et al. 2016) virtually to zero, but it will not significantly alter the merger rate of $\mathrm{BH}$ binaries formed dynamically in NSCs unless the birth kick velocities are $\gg 100 \mathrm{~km} \mathrm{~s}^{-1}$. As we will show in Section 4.2, these basic predictions are in agreement with the results of our cluster models; for now, we note that the obvious consequence of the comparison shown in Figure 1 is that the NSC versus the GC relative contribution to the $\mathrm{BH}$ merger rate will depend on the poorly constrained natal kick velocity distribution. In the following, we assume that at least some BHs are retained inside the cluster and consider the subsequent formation and dynamical evolution of binary BHs.

\subsection{Mass Segregation}

After a few million years from the birth of a star cluster, the most massive stars explode in supernovae or collapse directly to form BHs. If the BHs are not ejected by their natal kicks, being more massive than a typical main-sequence star, they will migrate to the cluster center via dynamical friction in a process that is generally referred to as mass segregation. In the dense environment of the cluster core BHs can efficiently form binaries, which will then harden and eventually merge. A useful reference time is the two-body relaxation timescale evaluated at the half-mass radius of the star cluster (Spitzer 1987):

$$
t_{\mathrm{rh}} \approx 4.2 \times 10^{9}\left(\frac{15}{\ln \Lambda}\right)\left(\frac{r_{\mathrm{h}}}{4 \mathrm{pc}}\right)^{3 / 2}\left(\frac{M_{\mathrm{cl}}}{10^{7} M_{\odot}}\right)^{1 / 2} \text { years }
$$

with $\ln \Lambda$, the Coulomb logarithm. On a time $t_{\text {rh }}$, two-body gravitational interactions of stars are important in driving the dynamical evolution of the cluster.

While low mass NSCs have short relaxation times, for some of the most massive NSCs, the half-mass relaxation time can exceed the Hubble time. However, even in the most massive NSCs, the BHs can still segregate at the center on the much shorter dynamical friction timescale (Chandrasekhar 1943). More precisely, the BHs will decay to the cluster core on the timescale (e.g., Binney \& Tremaine 1987):

$$
t_{\mathrm{df}} \approx 0.42 \times 10^{9}\left(10 \frac{m_{\star}}{m_{\bullet}}\right)\left(\frac{t_{\mathrm{rh}}}{4.2 \times 10^{9} \text { years }}\right) \text { years }
$$

with $m_{\star}$ and $m_{\bullet}$, the mass of a typical cluster star and $\mathrm{BH}$ respectively. After a time $t_{\mathrm{df}}$, the $\mathrm{BHs}$ will dominate the densities inside the cluster core. ${ }^{1}$

In Figure 2, we plot the half-mass radius (or effective radius) versus the total stellar mass for various types of compact clusters: NSCs, GCs, and Ultra Compact Dwarfs (UCDs). The dashed line delineates the region below which the dynamical friction timescale becomes shorter than $\approx 5$ Gyr suggesting that the BHs in these systems will sink to the center in much less than a Hubble time. Virtually all systems we considered except for the most massive NSCs and UCDs $\left(M_{\mathrm{cl}} \gtrsim 10^{8} M_{\odot}\right)$ can evolve rapidly enough so that the $\mathrm{BHs}$ will sink to the center, where they can participate in dynamical interactions and swap into hard binaries. The formation of such binaries and their dynamical evolution is discussed in the following.

\subsection{Formation of BH Binaries, Hardening, and Mergers}

After the BHs segregate to the cluster core, BH-binary formation can efficiently occur through the processes described below.

During core-collapse, if the densities of BHs become sufficiently high, BH binaries can be assembled through three-body processes in which a binary is formed with the help of a third $\mathrm{BH}$, which carries away the excess energy needed to bound the pair (Lee 1995; Morscher et al. 2015). The timescale for three-body binary formation can be written as

\footnotetext{
1 Note that Equations (2) and (3) are strictly valid only for a singular isothermal sphere model.
} 


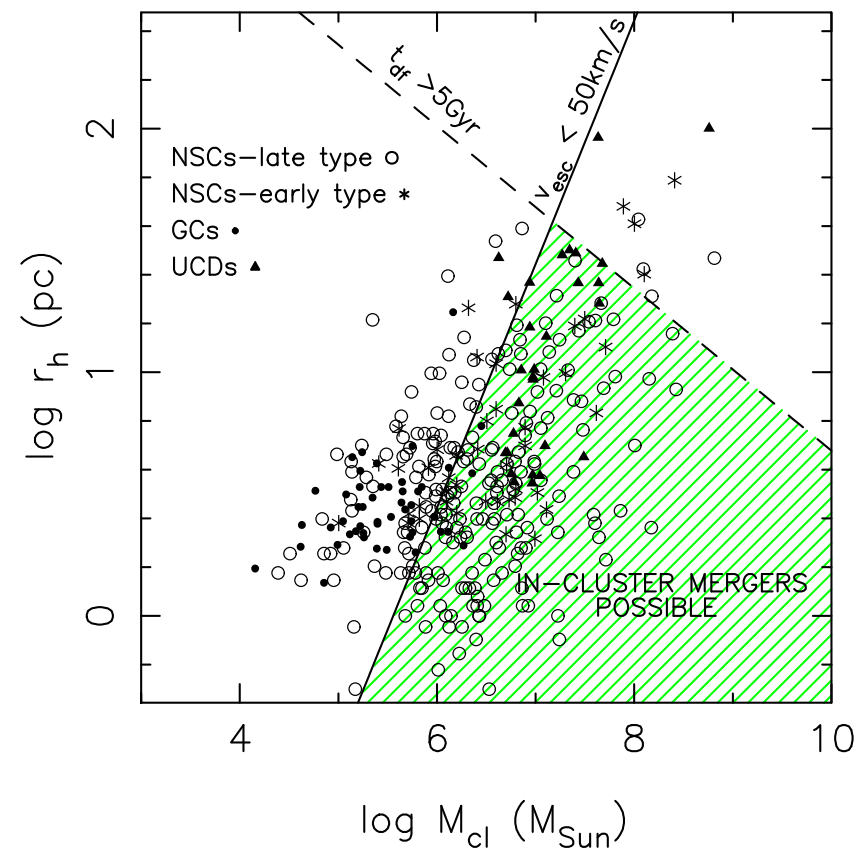

Figure 2. Half-mass radius (or effective radius) against total cluster mass for NSCs, GCs, and UCDs. Data are from Côté et al. (2006) and Georgiev et al (2016). Systems that lie to the right of the dashed line have $t_{\mathrm{df}}>5 \mathrm{Gyr}$. Such systems are still in the process of forming a BH subsystem. For the majority of the systems, we considered, including most NSCs and UCDs, the bulk of the stellar-mass BHs are likely to have already experienced significant mass segregation. Systems that lie to the left the solid black line have $v_{\text {esc }}<50 \mathrm{~km} \mathrm{~s}^{-1}$. This is an indicative value of cluster escape velocity below which $\mathrm{BH}$ binaries will be ejected from the cluster before merging. In systems that are within the green hatched region, the BHs are more likely to merge while still inside their host cluster.

(e.g., Lee 1995)

$$
\begin{aligned}
t_{3 \mathrm{bb}} \approx & 4 \times 10^{9}\left(\frac{n}{10^{6} \mathrm{pc}^{-3}}\right)^{-2}\left(\zeta^{-1} \frac{\sigma}{30 \mathrm{~km} \mathrm{~s}^{-1}}\right)^{9} \\
& \times\left(\frac{m_{\star}}{m \cdot} 10\right)^{9 / 2}\left(\frac{m \cdot}{10 M_{\odot}}\right)^{-5} \text { years, }
\end{aligned}
$$

with $n$ the number density of black holes near the center. The constant $\zeta \leqslant 1$ in the previous expression parametrizes the departure of the cluster from equipartition and we have used the relation $m \cdot\left(\zeta \sigma_{\mathrm{BH}}\right)^{2}=m_{\star} \sigma^{2}$ in order to express $t_{3 \mathrm{bb}}$ in terms of the cluster stellar velocity dispersion.

In addition to three-body binary formation, $\mathrm{BH}$ binaries can potentially form through exchange interactions involving primordial stellar binaries (Miller \& Lauburg 2009). Exchange interactions can lead to the efficient formation of $\mathrm{BH}$ binaries only if the cluster contains a number of hard binaries. Thus, this channel might be somewhat suppressed in NSCs-because of the larger velocity dispersion than in GCs a larger fraction of binaries will be soft and will be quickly ionized in NSCs. However, as argued in Miller \& Lauburg (2009), the reduction is not going to be by a large fraction given that binaries are typically born with roughly equal probability per logarithmic interval of semimajor axis, $d P / d \log (a)=$ const., in the range of $10^{-2}-10^{3}$ au (Duquennoy \& Mayor 1991). If, for example, we consider an NSC with velocity dispersion $\approx 30 \mathrm{~km} \mathrm{~s}^{-1}$ all binaries with semimajor axes $a \lesssim 1$ au will be hard. If we assume a constant probability per $\log (a)$ for $0.01<a<10^{3} \mathrm{au}$, then the probability of finding a binary in the range of $a=0.01-1$ au is substantial, $\approx 40 \%$. When a $\mathrm{BH}$ gets within a couple of semimajor axes lengths of a binary, the binary will be broken apart and the $\mathrm{BH}$ will tend to acquire a companion. The characteristic timescale on which such an exchange interaction occurs is $t_{1-2}=(n \Sigma \sqrt{3} \sigma)^{-1}$, where $\Sigma=\pi r_{\mathrm{p}}^{2}\left[1+2 G m_{123} /(\sqrt{3} \sigma)^{2} r_{\mathrm{p}}\right]$ is the interaction cross section for periapsis distances $\leqslant r_{\mathrm{p}} \approx 2$ au and $m_{123}$ is the total mass of the interacting objects. If the cluster core is dominated by stellar binaries, then the timescale for a $\mathrm{BH}$ to be captured into a binary is (Miller \& Lauburg 2009)

$$
\begin{aligned}
t_{1-2} \approx & \times 10^{9}\left(\frac{f_{b}}{0.01}\right)^{-1}\left(\frac{n}{10^{6} \mathrm{pc}^{-3}}\right)^{-1}\left(\frac{\sigma}{30 \mathrm{~km} \mathrm{~s}^{-1}}\right) \\
& \times\left(\frac{m_{123}}{10 M_{\odot}}\right)^{-1}\left(\frac{a_{\text {hard }}}{1 \mathrm{au}}\right)^{-1} \text { years, }
\end{aligned}
$$

where $a_{\text {hard }}$ is the typical semimajor axis of hard binaries and $f_{b}$ is the core binary fraction. By comparing the previous equation to Equation (4), we see that even under quite standard conditions (but even more so during core-collapse), a threebody binary formation likely dominates the initial dynamical formation of BH binaries in NSCs (Morscher et al. 2015).

Two-body binary formation can also occur through gravitational bremsstrahlung in which two initially unbound $\mathrm{BHs}$ become bound after a close encounter in which energy is dissipated through gravitational wave radiation. If a $\mathrm{BH}$ binary is formed in this manner, it mergers almost immediately, without further interactions. However, Lee (1995) showed that for velocity dispersions $\sigma \lesssim 100 \mathrm{~km} \mathrm{~s}^{-1}$ and numbers of BHs $\lesssim 10^{3}$ expected in the most massive star clusters, we study here, the rate of binary formation from gravitational bremsstrahlung is much less than that of regular three-body binary formation. Therefore, for our investigation, we do not account for binary formation though gravitational bremsstrahlung, but we caution that this process could become important in the most massive NSCs. In addition, we assume that after $\mathrm{BH}$ binaries are formed, binary-single interactions dominate over binarybinary interactions, which will be the case unless the binary fraction is very high ( $\geqslant 50 \%$; Morscher et al. 2015).

After BH binaries are formed, they will dominate the dynamics inside the cluster core. Assuming the interaction is now between three BHs each with masses of $10 M_{\odot}$, the typical timescale on which a three-body interaction occurs is

$$
\begin{aligned}
t_{1-2} \approx & 3 \times 10^{8} \zeta^{-1}\left(\frac{f_{b}}{0.01}\right)^{-1}\left(\frac{n}{10^{6} \mathrm{pc}^{-3}}\right)^{-1} \\
& \times\left(\frac{\sigma}{30 \mathrm{~km} \mathrm{~s}^{-1}}\right)\left(\frac{m_{\star}}{m .} 10\right)^{1 / 2} \\
& \times\left(\frac{m_{123}}{30 M_{\odot}}\right)^{-1}\left(\frac{a_{\mathrm{hard}}}{1 \mathrm{au}}\right)^{-1} \text { years. }
\end{aligned}
$$

Given that three-body encounters tend to pair the most massive BHs participating in the interaction, we expect that after a time $\lesssim t_{1-2}$ the most massive BHs in the cluster will become part of a hard binary. 
After a hard binary is formed, it will tend to harden at a constant rate (e.g., Quinlan 1996)

$$
\left.\frac{d a}{d t}\right|_{\mathrm{dyn}}=-H \frac{G \rho}{\sigma} a^{2} .
$$

In this last expression, $\rho$ is the local density of stars and BHs, $H \approx 20$ is the binary hardening rate, and we have assumed all equal-mass interlopers.

If after a single interaction with a cluster member of mass $m$., the semimajor axis of the binary decreases from $a$ to $a_{\text {fin }}$, then a binary with components of mass $m_{1}$ and $m_{2}$ will recoil with a velocity of $v_{2-1}^{2}=G \mu \frac{m \cdot}{m_{123}}\left(1 / a_{\text {fin }}-1 / a\right) \approx 0.2 G \mu \frac{m \cdot}{m_{123}} q_{3} / a$, where $\mu=m_{1} m_{2} / m_{12}, \quad m_{12}=m_{1}+m_{2}, \quad m_{123}=m_{1}+m_{2}+$ $m$. and $q_{3}=m_{0} / m_{12}$. In deriving the previous expression, we have assumed that in the interaction the binding energy of the binary increases by a fraction of $\approx 0.2 q_{3}$ (Quinlan 1996). The previous expressions can be used to derive the limiting semimajor axis below which a three-body interaction will eject the binary from the system:

$$
\begin{aligned}
a_{\mathrm{ej}} & =0.2 G \mu \frac{m \bullet}{m_{123}} q_{3} / v_{\mathrm{esc}}^{2} \\
& =0.07\left(\mu \frac{m \bullet}{m_{123}} q_{3} \frac{1}{M_{\odot}}\right)\left(\frac{v_{\mathrm{esc}}}{50 \mathrm{~km} \mathrm{~s}^{-1}}\right)^{-2} \mathrm{au} .
\end{aligned}
$$

The binary keeps hardening at a constant rate until either $\mathrm{GW}$ radiation takes over and drives its merger or it is ejected from the cluster. The time evolution of the binary semimajor axis due to $\mathrm{GW}$ radiation is described by the orbit averaged evolution equation (Peters 1964):

$$
\begin{aligned}
\left.\frac{d a}{d t}\right|_{\mathrm{GW}}= & -\frac{64}{5} \frac{G^{3} m_{1} m_{2} m_{12}}{c^{5} a^{3}\left(1-e^{2}\right)^{7 / 2}} \\
& \times\left(1+\frac{73}{24} e^{2}+\frac{37}{96} e^{4}\right),
\end{aligned}
$$

where $e$ is the binary eccentricity. The merger time for the two $\mathrm{BHs}$ is

$$
\begin{aligned}
t_{\mathrm{GW}} \approx & 2 \times 10^{9}\left(\frac{m_{1} m_{2} m_{12}}{10^{3} M_{\odot}^{3}}\right)^{-1}\left(\frac{a}{0.05 \mathrm{au}}\right)^{4} \\
& \times\left(1-e^{2}\right)^{7 / 2} \text { years. }
\end{aligned}
$$

Comparing the above expression with the expression for $a_{\mathrm{ej}}$ demonstrates an important point: since the larger the escape velocity from the cluster the smaller $a_{\mathrm{ej}}, \mathrm{BH}$ binaries that are produced in NSCs will have shorter merger times and are therefore more likely to merge within one Hubble time than $\mathrm{BH}$ binaries from lower mass GCs.

Let $a_{\mathrm{GW}}$ be the semimajor axis at which GW radiation begins to dominate the energy loss from the binary. A reasonable choice is to set $a_{\mathrm{GW}}$ equal to the semimajor axis at which $d a /\left.d t\right|_{\mathrm{dyn}}=d a /\left.d t\right|_{\mathrm{GW}}$. Assuming a circular binary, this leads to the relation (e.g., Merritt 2013)

$$
\begin{aligned}
a_{\mathrm{GW}}= & 0.05\left(\frac{m_{12}}{20 M_{\odot}}\right)^{3 / 5}\left(\frac{q}{(1+q)^{2}}\right)^{1 / 5} \\
& \times\left(\frac{\sigma}{30 \mathrm{~km} \mathrm{~s}^{-1}}\right)^{1 / 5}\left(\frac{10^{6} M_{\odot} \mathrm{pc}^{-3}}{\rho}\right)^{1 / 5} \mathrm{au},
\end{aligned}
$$

where $q=m_{2} / m_{1} \lesssim 1$. If $a_{\mathrm{GW}}>a_{\mathrm{ej}}$ merger happens before ejection. By comparing Equation (11) with the expression for $a_{\mathrm{ej}}$, we see that BH binaries that are produced in NSCs are less likely to be ejected from the cluster.

The binary will continue to interact with other cluster members until it reaches a semimajor axis $a_{\text {crit }}=$ $\max \left(a_{\mathrm{GW}}, a_{\mathrm{ej}}\right)$. After the binary has decayed to $a_{\text {crit }}$ (where it spends most of its lifetime), the timescale between two consecutive interactions becomes (e.g., Gültekin et al. 2004)

$$
\begin{aligned}
t_{2-1} \approx & 2 \times 10^{7} \zeta^{-1}\left(\frac{n}{10^{6} \mathrm{pc}^{-3}}\right)^{-1}\left(\frac{\sigma}{30 \mathrm{~km} \mathrm{~s}^{-1}}\right) \\
& \times\left(\frac{m_{\star}}{m .} 10\right)^{1 / 2}\left(\frac{a_{\text {crit }}}{0.05 \mathrm{au}}\right)^{-1}\left(\frac{m_{12}}{20 M_{\odot}}\right)^{-1} \text { years. }
\end{aligned}
$$

If we assume, as before, that each interaction removes a fraction $0.2 q_{3}$ of the binary binding energy (Quinlan 1996), then the timescale required to decay to $a_{\text {crit }}$ from a much larger separation is of the order of (Miller \& Hamilton 2002)

$$
t_{\text {merge }} \approx 5 q_{3}^{-1} t_{2-1} \text {. }
$$

During the hard interaction, the interloper will recoil at a speed of $v_{3}=v_{2-1} / q_{3}$. Thus the field BHs will start being ejected when $v_{3} \gtrsim v_{\text {esc }}$; at this point, the binary semimajor axis is $a_{3}=a_{\mathrm{ej}} / q_{3}^{2}$. At a fractional hardening of $\approx 0.2 q_{3}$ per interaction, the mass ejected from the cluster required in order to shrink the binary semimajor axis from $a_{3}$ to $a_{\text {crit }}$ is approximately

$$
M_{\mathrm{ej}} \approx m_{12} \ln \left(\frac{a_{3}}{a_{\text {crit }}}\right),
$$

so that for low mass clusters $M_{\mathrm{ej}} \approx m_{12} \ln \left(1 / q_{3^{2}}\right)$ and for high mass clusters $M_{\mathrm{ej}} \approx m_{12} \ln \left(a_{\mathrm{ej}} / a_{\mathrm{GW}} q_{3}^{2}\right)$. Given that $a_{\mathrm{ej}}$ decreases with the cluster escape speed, the previous equation implies that the larger the cluster mass is, the fewer $\mathrm{BH}$ interlopers will be ejected in addition to fewer binaries being ejected; when

$$
v_{\mathrm{esc}} \gtrsim 120\left(\mu \frac{m_{12}}{m_{123}} \frac{4}{M_{\odot}}\right)^{1 / 2}\left(\frac{a_{\mathrm{GW}}}{0.05 \mathrm{au}}\right)^{-1 / 2} \mathrm{~km} \mathrm{~s}^{-1}
$$

the $\mathrm{BH}$ binary will merge without ejecting any of the field BHs.

From the condition $a_{\mathrm{ej}}<a_{\mathrm{GW}}$, we derive the critical cluster escape velocity above which binaries will merge before being ejected through hard scattering with surrounding stars:

$$
\begin{aligned}
\tilde{v}_{\mathrm{esc}} \gtrsim & 110 \frac{\sqrt{q}}{1+q}\left(\frac{m \bullet}{10 M_{\odot}}\right) \\
& \times\left(\frac{30 M_{\odot}}{m_{123}}\right)^{1 / 2}\left(\frac{a_{\mathrm{GW}}}{0.05 \mathrm{au}}\right)^{-1 / 2} \mathrm{~km} \mathrm{~s}^{-1},
\end{aligned}
$$

so that for $m_{1}=m_{2}=m_{\bullet}=10 M_{\odot}$, we have $\tilde{v}_{\mathrm{esc}} \approx 50 \mathrm{~km} \mathrm{~s}^{-1}$. The solid line in Figure 2 shows the locus of points where the escape velocity from the clusters, $v_{\mathrm{esc}}\left(\mathrm{km} \mathrm{s}^{-1}\right) \approx$ $0.1 \sqrt{M_{\mathrm{cl}}\left(M_{\odot}\right) / r_{\mathrm{h}}(\mathrm{pc})}$ (see Equation (1) above), is equal to $50 \mathrm{~km} \mathrm{~s}^{-1}$. The $\mathrm{BH}$ binaries forming in clusters lying to the left of this line are likely to be ejected before the merger. The approximate relation $v_{\mathrm{esc}} \approx 2 \sqrt{3} \sigma$ implies that only clusters with velocity dispersions of $\sigma \gtrsim 15 \mathrm{~km} \mathrm{~s}^{-1}$ will be able to 
retain their binaries. In many NSCs and UCDs, stellar-mass BH binaries will merge while still inside the cluster, while most $\mathrm{BH}$ mergers in GCs are expected to occur outside the cluster unless an initially already massive $\mathrm{BH}\left(M \gtrsim 100 M_{\odot}\right)$ is present in the system (Gültekin et al. 2006). This result is consistent with Monte Carlo simulations of GC models, where the vast majority of $\mathrm{BH}$ binaries assembled dynamically through $\mathrm{N}$ body interactions are found to merge after escaping from their host systems (e.g., Downing et al. 2011).

Figure 2 shows that, in many NSCs and UCDs, the $\mathrm{BH}$ merger remnants are likely to be retained so they might form new $\mathrm{BH}$ binaries that will subsequently harden and merge. It is therefore possible that $\mathrm{BHs}$ in these massive star clusters will undergo a number of repeated mergers and grow considerably.

In addition to the recoil kick due to three-body interactions, as two compact objects merge, asymmetric emission of gravitational radiation will also induce a recoil velocity that can eject the merger product from the system. In the next section, we discuss this additional effect.

\subsection{GW Recoil}

The GW recoil velocity of a merged $\mathrm{BH}$ depends on the mass ratio and spins of the progenitor BHs. Hence, in order to make predictions about the distribution of recoil velocities for dynamically formed BHs in star clusters, we first define the pre-merger $\mathrm{BH}$ spin and mass distributions.

The mass distribution of $\mathrm{BH}$ binaries is quite uncertain. Here, we use the $\mathrm{BH}$ mass distribution of the dynamically formed merging black hole binaries from the Monte Carlo models of massive star clusters presented in Chatterjee et al. (2016). These distributions contain no BH binaries with mass ratios less than $q \approx 0.5$. This is expected given that dynamical encounters in star clusters tend to pair and eject tight $\mathrm{BH}$ binaries with similar mass components-binaries in dense stellar environments are prone to exchange components, preferentially ejecting lighter partners in favor of more massive companions (Sigurdsson \& Hernquist 1993).

The distribution of $\mathrm{BH}$ spins is also very uncertain. If the $\mathrm{BH}$ inspiral is driven predominantly by random gravitational interactions with other BHs and stars, we might expect the spin orientations to be close to random. We note that if there is significant coherent gas accretion, the spins might align in a way that might lead to low recoil kicks (e.g., Bogdanović et al. 2007). However, stellar-mass BHs are unlikely to accrete enough mass from the interstellar medium for this process to be effective (Miller \& Lauburg 2009). For these reasons, in our computations, the misalignment angle of each $\mathrm{BH}$ is chosen at random in $\cos (\theta)$.

For the spin magnitudes of the BHs, we consider two choices. The blue solid line in the left panel of Figure 3 corresponds to a "uniform" model in which the initial spin magnitudes are drawn uniformly from the range $\chi=[0,1)$, where $\chi$ is the dimensionless spin of the BH $\left(\chi=S / m_{.}^{2}\right.$, where $\boldsymbol{S}$ is the spin angular momentum in units of $m^{2}$ ). The blue dashed line corresponds to one additional "high spin" model in which the spin magnitude is set to a fixed value, $\chi=0.9$. We note here that our spin magnitude distributions differ, for example, from those of O'Leary et al. (2016) who adopted low-spinning BHs, leading to low merger kick velocities. Our choice is motivated by observations: typical estimates of stellar-mass $\mathrm{BH}$ spins suggest high values, $\chi>0.5$, in many cases (for a review, see Miller \& Miller 2015). In addition, equal-mass non-spinning binaries produce a rotating (Kerr) $\mathrm{BH}$ with a final spin magnitude of $\chi \approx 0.69$ (Hofmann et al. 2016), so that BHs undergoing more than one merger inside the cluster will have a finite spin magnitude (Berti et al. 2007). However, we note that configurations leading to rapidly spinning $\mathrm{BHs}$ are rare. The dimensionless spin magnitude tends to decrease for a $\mathrm{BH}$ that engages in a series of mergers, if the lighter BHs with which it mergers have a constant mass (Miller 2002; Blandford \& Hughes 2003). This will keep the growing BH safely in the cluster after the first few mergers: not only does the mass ratio get farther from unity, which decreases the kick, but the spin of the more $\mathrm{MBH}$ drops as well.

After the pre-merger $\mathrm{BH}$ spin and mass distributions have been defined, we compute the recoil kick velocity from the following fitting formula based on the results from numerical relativity simulations of Lousto et al. (2012):

$$
\begin{gathered}
\boldsymbol{v}_{\mathrm{k}}=v_{\mathrm{m}} \hat{\boldsymbol{e}}_{\perp, 1}+v_{\perp}\left(\cos \xi \hat{e}_{\perp, 1}+\sin \xi \hat{\boldsymbol{e}}_{\perp, 2}\right)+v_{\|} \hat{\boldsymbol{e}}_{\|}, \\
v_{\mathrm{m}}=A \eta^{2} \sqrt{1-4 \eta}(1+B \eta), \\
v_{\perp}=\frac{H \eta^{2}}{(1+q)}\left(\chi_{2 \|}-q \chi_{1 \|}\right), \\
v_{\|}=\frac{16 \eta^{2}}{(1+q)}\left[V_{1,1}+V_{\mathrm{A}} \tilde{S}_{\|}+V_{\mathrm{B}} \tilde{S}_{\|}^{2}+V_{\mathrm{C}} \tilde{S}_{\|}^{3}\right] \\
\quad \times\left|\chi_{2 \perp}-q \chi_{1 \perp}\right| \cos \left(\phi_{\Delta}-\phi_{1}\right),
\end{gathered}
$$

where $\eta \equiv q /(1+q)^{2}$ is the symmetric mass ratio, $\perp$ and $\|$ refer to vector components perpendicular and parallel to the orbital angular momentum, respectively, $\hat{e}_{\perp, 1}$ and $\hat{e}_{\perp, 2}$ are orthogonal unit vectors in the orbital plane, and $\tilde{\boldsymbol{S}} \equiv$ $2\left(\chi_{2}+q^{2} \chi_{1}\right) /(1+q)^{2}$. The values of $A=1.2 \times 10^{4} \mathrm{~km}$ $\mathrm{s}^{-1}, B=-0.93, H=6.9 \times 10^{3} \mathrm{~km} \mathrm{~s}^{-1}$, and $\xi=145^{\circ}$ are from González et al. (2007) and Lousto \& Zlochower (2008), and $V_{1,1}=3678 \mathrm{~km} \mathrm{~s}^{-1}, V_{\mathrm{A}}=2481 \mathrm{~km} \mathrm{~s}^{-1}, V_{\mathrm{B}}=1793 \mathrm{~km}$ $\mathrm{s}^{-1}$, and $V_{\mathrm{C}}=1507 \mathrm{~km} \mathrm{~s}^{-1}$ are taken from Lousto et al. (2012). The angle $\phi_{\Delta}$ is that between the in-plane component $\Delta_{\perp}$ of the vector $\boldsymbol{\Delta} \equiv M^{2}\left(\chi_{2}-q \chi_{1}\right) /(1+q)$ and the infall direction at merger. We take the phase angle $\phi_{1}$ of the binary to be random.

The histograms in the left panel of Figure 3 show the escape velocities from NSCs and GCs computed as described in Section 2.1. The blue curves show the recoil velocity distributions for our models computed using Equation (17). The recoil velocity distribution in the uniform spin model is peaked at $v_{\mathrm{k}} \approx 500 \mathrm{~km} \mathrm{~s}^{-1}$, while the high spin model produces significantly larger kicks with typical velocities of $v_{\mathrm{k}} \approx 1000 \mathrm{~km} \mathrm{~s}^{-1}$. Note, however, that in both models there is a substantial fraction of systems that are accelerated with velocities $\lesssim 100 \mathrm{~km} \mathrm{~s}^{-1}$

The left panel of Figure 3 suggests that only the most massive GCs have a finite probability of retaining a $\mathrm{BH}$ merger remnant formed inside the cluster. Considering also that $\mathrm{BH}$ binaries in GCs are likely to be flung before merger due to three-body encounters, we conclude that the retention probability of BH merger remnants in GCs is small. The left panel of Figure 3 shows instead that the escape velocities of many NSCs are high enough that a substantial number of mergers are expected to be retained inside these systems. 

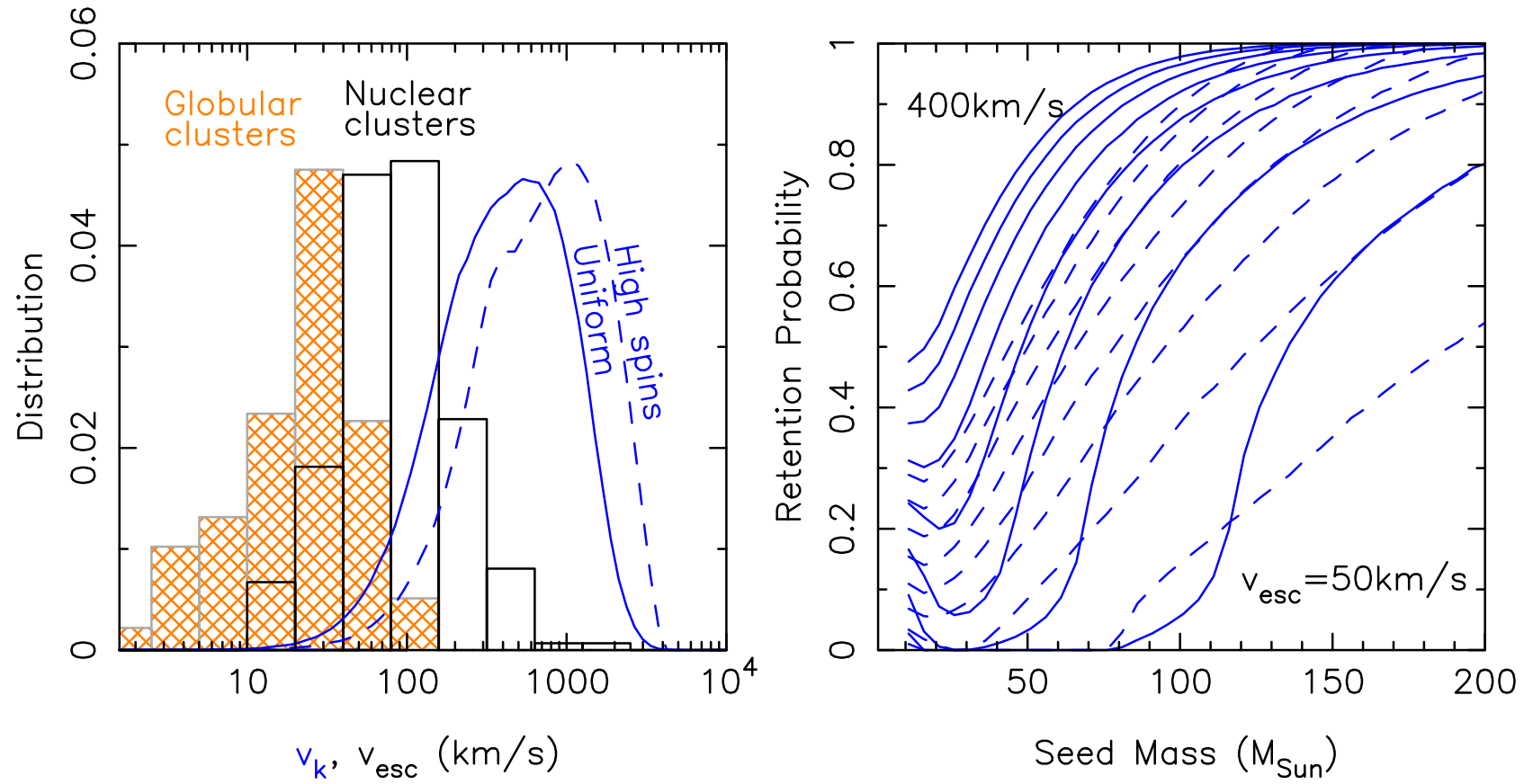

Figure 3. Left panel: distribution of escape velocities from NSCs and GCs (histograms) compared to the distributions of GW kick velocities of merging BHs (blue curves). The solid blue line corresponds to a model in which the spin magnitude was chosen randomly in the range $\chi=[0,1)$; the dashed blue line corresponds to a high spin model in which $\chi=0$.9. Right panel: probability of remaining inside the cluster as a function of initial mass of the dominant $\mathrm{BH}$ and for different values of the cluster escape velocity. Here we have assumed that the mass of the secondary BH is $10 M_{\odot}$. The solid line is for the uniform spin model; the dashed line is for the high spin model. These plots show that the recoil velocity imparted by the anisotropic emission of GW radiation will lead to the ejection of most BH merger remnants formed inside GCs, while in NSCs a fraction of BHs will be retained. For this reason, BHs of large mass can naturally grow inside NSCs through the repeated accretion of lower mass BHs. Thus, NSCs are a likely host environment for the highest-mass BH mergers that are potentially detectable by aLIGO.

In the right panel of Figure 3, we compute the probability of remaining in the cluster for our spin distributions as a function of the initial $\mathrm{BH}$ mass and assuming that the secondary $\mathrm{BH}$ mass is $10 M_{\odot}$. For escape velocities $\lesssim 50 \mathrm{~km} \mathrm{~s}^{-1}$ (typical of massive GCs), the probability of remaining inside the cluster after a merger is essentially zero, unless the cluster contains initially a BH seed of mass $\gtrsim 100 M_{\odot}$. For escape velocities of $200 \mathrm{~km} \mathrm{~s}^{-1}$, which are more typical of NSCs, the probability of retaining a BH merger remnant with a initial mass of $50 M_{\odot}$ is approximately 0.5 or 0.3 depending on the assumed spin distribution. This makes NSCs excellent candidates for producing massive $\mathrm{BH}$ mergers that are potentially observable by aLIGO, because they can retain their $\mathrm{BHs}$, while also evolving rapidly enough that the BHs can sink back to the center and dynamically form new binaries, which will subsequently merge. This merger channel is expected to occur quite naturally in massive stellar clusters, such as NSCs and UCDs, while it is unlikely to happen in lower mass systems such as open clusters and GCs.

In the next section, we present a semi-analytical model that we use in order to make predictions about the mass distribution and rates of BH-binary mergers forming in NSCs.

\section{SEMI-ANALYTICAL MODELING}

As argued above, the dynamical evolution of NSCs is of great interest because these systems could represent a important source of inspiraling BHs detectable by aLIGO. However, a good understanding of the dynamical evolution of massive clusters $\left(M_{\mathrm{cl}} \gg 10^{6} M_{\odot}\right)$ and their implications for aLIGO detections is still elusive. The main difficulty is the large number of particles comprising these systems which makes their treatment extremely challenging even for approximate Monte Carlo methods. Here we adopt a semi-analytical approach, which allows us to make predictions about the expected rate and properties of inspiraling $\mathrm{BH}$ binaries forming in NSCs.

\subsection{Simplified Approach}

First, we define the structural properties of our star clusters. We assign a total stellar mass $M_{\mathrm{cl}}$ to the cluster. For $M_{\mathrm{cl}} \leqslant 5 \times 10^{6} M_{\odot}$, the half-mass radius is independent of the cluster mass and it is set to $r_{h}=3 \mathrm{pc}$. In the NSC mass regime, $M_{\mathrm{cl}}>5 \times 10^{6} M_{\odot}$, we adopt the fitted relation to the NSCs in late-type galaxies from Georgiev et al. (2016): $\log \left(r_{h} / c 1\right)=\alpha \log \left(M_{\mathrm{cl}} / c 2\right)+\beta$, with $\alpha=0.321$, $\beta=-0.011, \quad c 1=3.31 \mathrm{pc}$, and $c 2=3.6 \times 10^{6} M_{\odot}$. While sampling from the adopted distributions, we also accounted for the scatter of the observed relations. The escape velocity from the cluster is then computed using the approximate Equation (1) above; the cluster velocity dispersion is $\sigma=v_{\text {esc }} /(2 \sqrt{3})$. The central number density of stars was computed as $n=4 \times 10^{6}\left(\sigma / 100 \mathrm{~km} \mathrm{~s}^{-1}\right)^{2} \mathrm{pc}^{-3}$. This latter expression gives a central number density of stars for a MilkyWay-like NSC of $4 \times 10^{6} \mathrm{pc}^{-3}$ and $\approx 10^{5} \mathrm{pc}^{-3}$ for a $10^{6} M_{\odot}$ GC-this is consistent with observed values (Harris 1996; Merritt 2010).

Next, we define the initial mass distribution and number density of BHs in our cluster models. We take the mass distribution of single BHs from Figure 6 of Rodriguez et al. (2016a). These authors used the stellar evolution code BSE (Hurley et al. 2002, 2007) improved with the stellar remnant prescription from Kiel \& Hurley (2009) and Chatterjee et al. 
(2010). Our models adopt the update prescriptions for stellar winds and supernova fallback, in order to replicate the $\mathrm{BH}$ mass distribution of Dominik et al. (2013) and Belczynski et al. (2010). We consider two values of metallicity, $Z=0.01 Z_{\odot}$ and $Z=0.25 Z_{\odot}$, defined below as low metallicity and high metallicity models.

In our calculations, we assume that all clusters formed 12 Gyr ago regardless of their mass. While this is a good approximation for GCs, NSCs are known to have complex star formation histories, including recent episodes of star formation. We neglect such complications in the following, noting that the bulk of the stellar population in NSCs is also likely to be in old stars formed many gigayears ago (e.g., Pfuhl et al. 2011).

Initially, our cluster models have a total mass in BHs that is $M .=0.01 M_{\mathrm{cl}}$. This is the typical mass fraction in $\mathrm{BHs}$ expected for standard initial mass functions (e.g., Hopman \& Alexander 2006). The total number of BHs is therefore $N_{\bullet} \approx M_{\bullet} /\left\langle m_{\bullet}\right\rangle$, with $\left\langle m_{\bullet}\right\rangle$ the average BH mass in our models. Then, we consider natal kicks. For each $\mathrm{BH}$ in our fiducial model, we compute a natal kick velocity from a Maxwellian given by $\sigma_{\text {natal }}=265 \mathrm{~km} \mathrm{~s}^{-1}$, as commonly done for neutron stars (Hobbs et al. 2005), and assume that the natal velocity of a $\mathrm{BH}$ of mass $m$. is lowered by the factor of $1.4 M_{\odot} / m_{\text {. }}$. For any sufficiently massive $\mathrm{BH}$ progenitors $\left(>40 M_{\odot}\right)$, the fallback completely damps any natal kick, and the $\mathrm{BH}$ is retained in the cluster (Fryer \& Kalogera 2001). BHs that receive a kick with velocities larger than the escape velocity from the cluster are removed from our models. However, since we only consider massive clusters with large escape velocities, a large fraction of $\mathrm{BHs}$ in our models is retained after experiencing a natal kick. This makes our conclusions less sensitive to the prescription we used for natal kicks, provided that the real kick magnitudes are not much larger than what we have adopted here. We discuss in more detail the effect of varying the natal kick magnitudes in Section 4.2.

We assume that after a time $t_{\mathrm{df}}\left(\left\langle m_{\bullet}\right\rangle\right)$ the BHs have segregated to the cluster center. After this time, due to the high densities in the core, $\mathrm{BH}$ binaries will efficiently form though three-body binary formation (Morscher et al. 2015) and possibly through exchange interactions with stellar binaries (Miller \& Lauburg 2009). Therefore, we assume that after a time $t_{\mathrm{df}}\left(\left\langle m_{\bullet}\right\rangle\right)$, a fraction $f_{\text {bin }}=0.01$ of the BHs end up in hard $\mathrm{BH}$ binaries. Although this fraction is quite uncertain, the value we adopted is typical for Monte Carlo models of massive star clusters with a low binary fraction (e.g., see Figure 6 in Morscher et al. 2015).

After the BHs have segregated to the center and we have assigned a fraction of them to be in BH binaries, we follow the evolution, ejection, and formation of new binaries, adopting the scheme described in what follows.

We divide the $\mathrm{BH}$ cluster in $N_{\mathrm{B}}=f_{\text {bin }} N_{\bullet} / 2$ sub-groups, each containing the same number of BHs. We find the two most massive BHs in each sub-group and assume that after a time $t_{1-2}$ they form a binary. Thus, we assume that each sub-group always contains one binary and that this binary is always composed of the two most massive BHs in the sub-group.

While each binary is assumed to evolve in the gravitational potential of the entire cluster the adopted numerical scheme allows us to simulate a scenario in which the number of $\mathrm{BH}$ binaries in the cluster is approximately constant with time. This, besides allowing us to greatly simplify our approach, appears to be reasonable when compared to the results of
Monte Carlo models of massive clusters (Morscher et al. 2015; Chatterjee et al. 2016). Moreover, we assume that the $\mathrm{BH}$ binaries are always composed of the two most MBHs in each sub-group because, during exchange encounters, lighter partners are more likely to be ejected. This favors the formation of high mass binaries with similar mass components (e.g., Chatterjee et al. 2016). In our calculation, we conservatively assume that the interactions occur between BHs and stellar binaries so that the timescale for binary formation is the longer timescale given by Equation (5). The binary fraction in evaluating $t_{1-2}$ was computed taking a primordial binary fraction of 0.2 and lowering this fraction by the number of soft binaries for a constant probability in $\log (a)$ (Duquennoy \& Mayor 1991).

Any binary forms with an initial semimajor axis $a_{\text {hard }}=1 /\left(\sigma / 30 \mathrm{~km} \mathrm{~s}^{-1}\right)^{2}$ au. Given the cluster velocity dispersion, its density and the mass of the binary, we compute (1) the semimajor axis, $a_{\mathrm{GW}}$, below which $\mathrm{GW}$ radiation will start to dominate (Equation (11)) and (2) the semimajor axis, $a_{\mathrm{ej}}$, at which the binary will be ejected as a consequence of threebody scatterings. If $a_{\mathrm{ej}}>a_{\mathrm{GW}}$, the binary will merge outside the cluster and will be ejected with a semimajor axis $\approx a_{\mathrm{ej}}$; in this case, we evaluate the timescale from the formation of the binary to its ejection, $t_{\mathrm{ej}}$, using Equation (13) so that the lifetime of the binary is $T=t_{\mathrm{ej}}+t_{\mathrm{GW}}\left(a=a_{\mathrm{ej}}\right)$. If $a_{\mathrm{ej}}<a_{\mathrm{GW}}$, the binary will merge inside the cluster; in this latter case, the total lifetime of the binary is $T=t_{\text {merge }}+t_{\mathrm{GW}}\left(a=a_{\mathrm{GW}}\right)$. In the previous expressions, the GW merger timescale, $t_{\mathrm{GW}}$, was computed by sampling the binary eccentricity from a thermal distribution $N \propto e^{2}$.

If the $\mathrm{BH}$ binary is ejected from the cluster, then after a time $t_{1-2}$ we form a new binary and, as before, we take its components to be the next two most massive BHs in the subgroup. Then the hardening timescale of the binary is evaluated as before and it is determined whether the new binary will merge inside the cluster, and, if it does, whether it will be retained inside the cluster after merging.

If the binary merges inside the cluster (i.e., $a_{\mathrm{ej}}<a_{\mathrm{GW}}$ ), we assign the two progenitor BHs a spin magnitude and orientation from the spin models described in Section 2.4 and compute the GW recoil speed through Equation (17). In order to account for the recoil kick due to the interaction with a third object, we compute a total kick velocity as $v_{\text {tot }}=\sqrt{v_{\mathrm{k}}^{2}+v_{2-1}^{2}}$, with $v_{2-1}$ computed as in Section 2.3 (note that $v_{\text {tot }} \approx v_{\mathrm{k}}$ typically). If $v_{\text {tot }}>v_{\text {esc }}$, the $\mathrm{BH}$ merger remnant is ejected from the cluster, otherwise it is retained.

If the $\mathrm{BH}$ merger remnant is ultimately retained inside the cluster, it will have another chance of interacting with new binaries and experiencing additional mergers. In this case, we place the $\mathrm{BH}$ remnant at a distance of $r_{\mathrm{h}}\left(v_{\mathrm{tot}} / v_{\mathrm{esc}}\right)^{2}$ from the center and evaluate the dynamical friction timescale for the $\mathrm{BH}$ to reach the cluster core through Equation (3). If $t_{\mathrm{df}}$ is greater than $10 \mathrm{Gyr}$, the $\mathrm{BH}$ is removed from the computation, otherwise after a time $t_{1-2}$ the $\mathrm{BH}$ forms a new binary with the next most massive $\mathrm{BH}$ in the sub-group. Then, the hardening timescale of the new binary is evaluated as before and it is determined whether the new binary will merge inside the cluster, and if it does whether it will be ejected from the cluster after the recoil due to anisotropic emission of $\mathrm{GW}$ radiation.

As the binary hardens, we calculate the number of field $\mathrm{BHs}$ that are ejected through three-body encounters as 


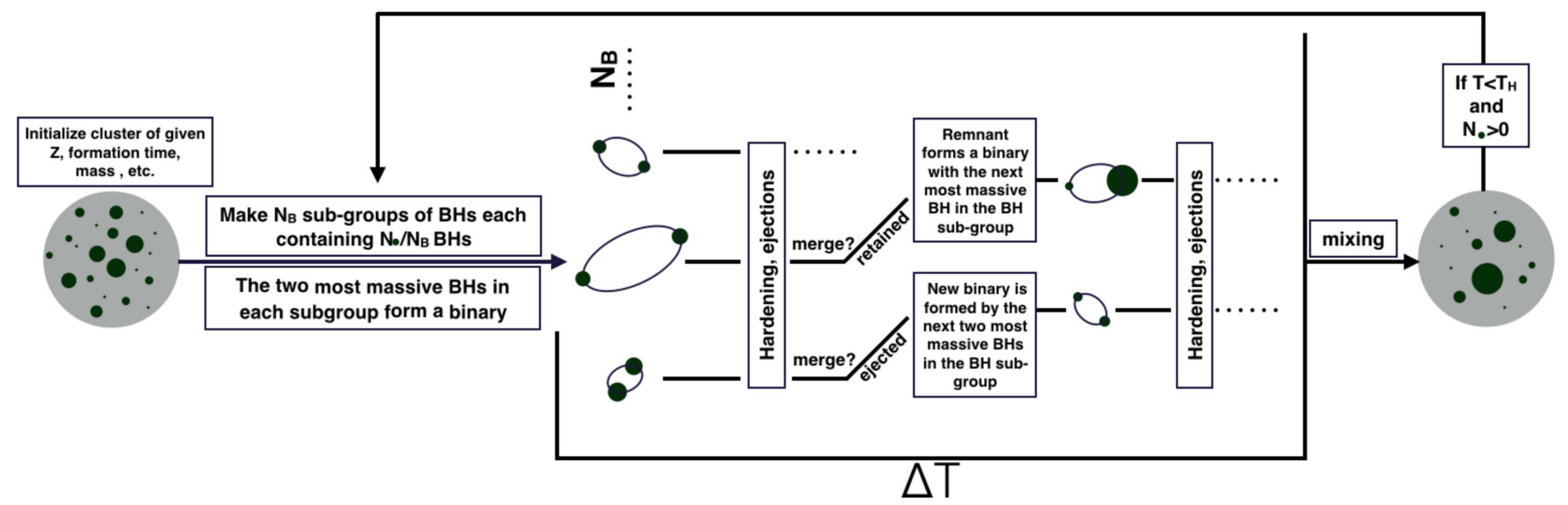

Figure 4. Schematic diagram illustrating our semi-analytical algorithm to model the evolution of BH binaries in stellar clusters. After we initialize the star cluster, we divide the BH population in sub-groups each containing an equal number of BHs. Each sub-group contains a BH binary for which the components are always the two BHs that are currently the two most massive in the sub-group. The binary is evolved for a time interval $\Delta t$; if the binary merges or is ejected from the cluster, a new binary is formed and evolved. After a time interval $\Delta t$, all BHs are mixed back together and the procedure is repeated until either all BHs have been ejected from the cluster or the integration time becomes longer then the Hubble time $\left(T_{\mathrm{H}}\right)$.

$N_{\mathrm{ej}}=M_{\mathrm{ej}} /\langle m$.$\rangle , where M_{\mathrm{ej}}$ is given by Equation (14). If the number of ejected $\mathrm{BHs}$ becomes larger than the total initial number of BHs, we stop the integration.

The previous steps (i.e., binary formation, hardening, and ejection/merger) are repeated for each sub-group for a time step $\Delta t$. After an interval of time $\Delta t$, the remaining $\mathrm{BHs}$ in all of the sub-groups are mixed together and the procedure described above is repeated recursively until either all BHs have been ejected from the cluster or the total integration time exceeds the Hubble time. The mixing of the sub-groups every $\Delta t$ allows us to avoid suppressing exchange interactions between massive $\mathrm{BHs}$ that might grow in different sub-groups. In what follows, we set $\Delta t=1.5 \times 10^{9}$ years, but found that values in the range of $\Delta t=1-3 \times 10^{9}$ years all produced similar results.

The main steps of our semi-analytical algorithm are also schematically illustrated in Figure 4 . We note that our prescriptions are oversimplified in many ways and that more accurate Monte Carlo simulations will be needed in order to confirm our results. One basic simplifying assumption is that the cluster structural properties (e.g., central density, half-mass radius) remain constant in time. We believe that this assumption is also be justified in many cases, especially in very massive clusters where the relaxation timescale is longer. For example, Monte Carlo simulations of moderately massive GCs find that $r_{\mathrm{h}}$ increases with time, but often only by a factor $\lesssim 3$ throughout the cluster evolution (Chatterjee et al. 2016). Additionally, in our models, we assume that the binary-single interaction rate is always dominant with respect to that of binary-binary interactions. This latter assumption is also reasonable, unless the cluster has a very large initial binary fraction ( $\gtrsim 0.5$; Morscher et al. 2015). Finally, we note that we do not follow the evolution of the $\mathrm{BH}$ spins through consecutive mergers but assume that the spins are always drawn from the assumed distributions.

\subsection{Results}

Given that our prescriptions are simplified in many ways, we proceed here by testing the results of our models against the results from the Monte Carlo models of Rodriguez et al. (2016a).
In the upper panel of Figure 5, we show the total number of mergers per cluster for systems containing different numbers of stars and having different metallicities. In order to convert $M_{\mathrm{cl}}$ in number of stars, we have taken a mean stellar mass of $0.55 M_{\odot}$ typical of old stellar populations (Merritt 2013). Moreover, we select here the BH spins based on the uniform spin model described in Section 2.4.

Our semi-analytical models predict that the total number of mergers increases with cluster mass and so do the Monte Carlo models. The total number of inspirals over $12 \mathrm{Gyr}$ is nearly linearly proportional to the final cluster mass. This result is also in agreement with previous models of GCs and shows that this statement can likely be extrapolated up to numbers of stars of the order of a few $10^{7} M_{\odot}$. Our models also predict an inversion of this simple correlation showing that for $M_{\mathrm{cl}} \gtrsim 10^{7} M_{\odot}$ the number of merging BHs flattens or even declines toward larger cluster masses. This is expected given that for such massive clusters with higher values of $\sigma$ have a larger binary formation time $t_{1-2}$. The most massive clusters in our integrations, which could represent NSCs, produce up to a few thousand $\mathrm{BH}$ mergers per cluster.

The lower panel of Figure 5 gives the median mass of the ejected $\mathrm{BH}$ binaries formed in 10 cluster models with mass $1.2 \times 10^{6} M_{\odot}$ and half-mass radius $r_{h}=7 \mathrm{pc}$. These results are directly compared to those from Figure 4 in Rodriguez et al. (2016a), which corresponds to a cluster model of initial mass $1.2 \times 10^{6} M_{\odot}$ and final half-mass radius $r_{h} \approx 7 \mathrm{pc}$. The good agreement between the results of our simplified approach and those of Monte Carlo simulations gives a high level of reliability to our semi-analytical models.

Figures 6 and 7 give the masses for each of the $\mathrm{BH}$ inspirals occurring in 10 star cluster models of metallicities $Z=0.01 Z_{\odot}$ and $Z=0.25 Z_{\odot}$ respectively. Masses in the ranges of $(0.5-5) \times 10^{6} M_{\odot}$ (upper panel) and $(0.5-5) \times 10^{7} M_{\odot}$ (lower panel) were considered. The overall structure of the plots agrees well with our understanding of the dynamics of BHs and their evolution in star clusters, and with the results of previous work (e.g., see Figure 7 of Rodriguez et al. 2016a). After the formation of the cluster at high redshift, the BHs segregate to the center, the most massive BHs form binaries and the majority of them are ejected. The cluster processes through its 

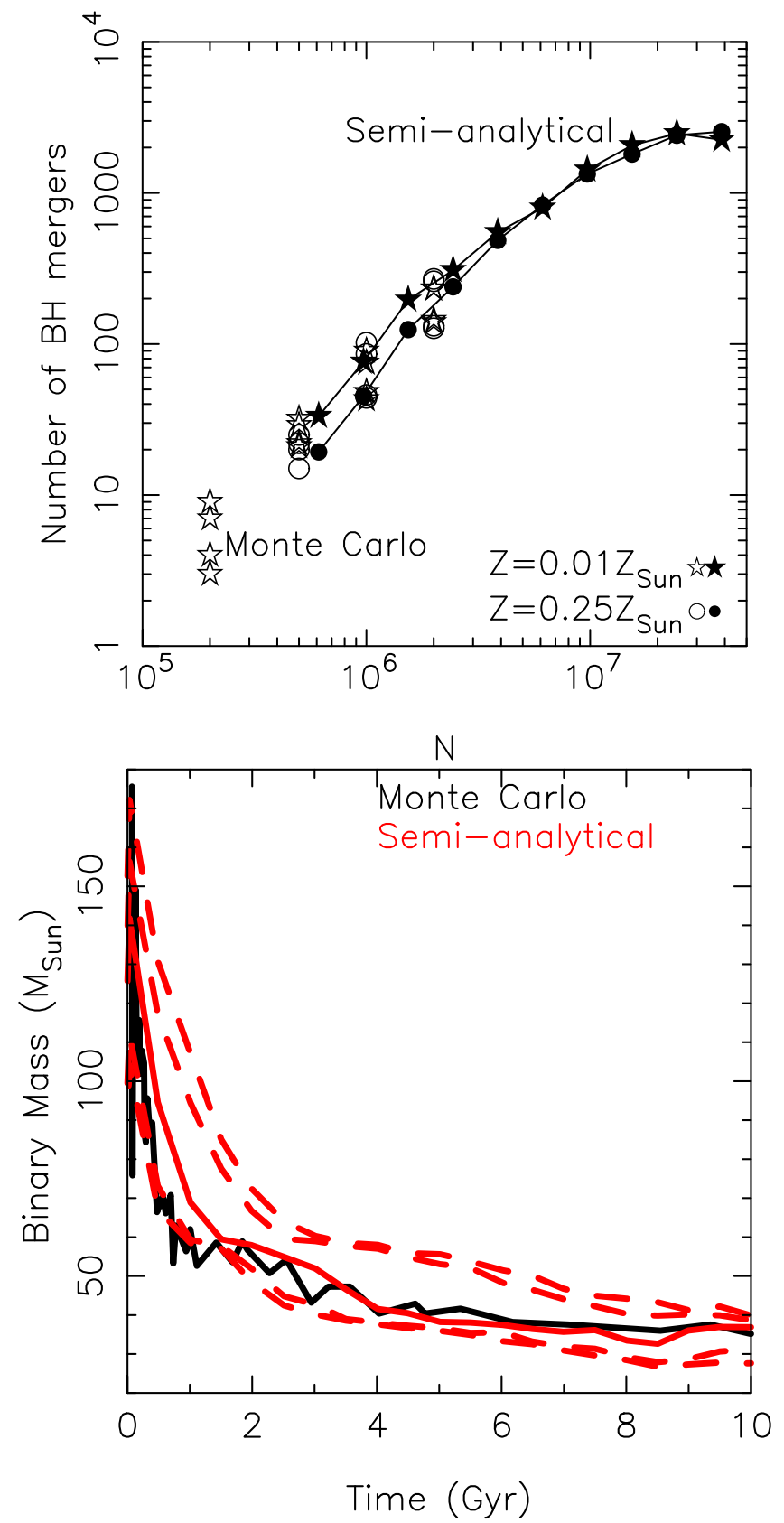

Figure 5. Comparison between the results of our semi-analytical model with the results of Monte Carlo models (Rodriguez et al. 2016a). The upper panel shows the median number of merging $\mathrm{BH}$ binaries as a function of the total number of stars in the cluster. Open symbols are from the Monte Carlo simulations of Rodriguez et al. (2016a). Filled symbols are from our simplified semi-analytical approach. The lower panel gives the median mass of ejected $\mathrm{BH}$ binaries as a function of time of ejection from a GC Monte Carlo model of Rodriguez et al. (2016a; black curve) and the average mass of ejected $\mathrm{BH}$ binaries from 10 semi-analytical models having similar structural properties (red curve). The dashed curves give the region containing $70 \%$ and $90 \%$ of the ejected systems in these models. Solid curve is the median of the mass distribution.

population of BHs that merge and are ejected from most to least massive, so that only low mass BHs are retained by the present epoch. More massive clusters, which could represent typical NSCs, produce BH mergers in the local universe that are significantly more massive than mergers occurring in lower mass clusters.
As also noted in Rodriguez et al. (2016a) the plateaus in the chirp mass and total mass distributions in Figure 7 are mainly a consequence of the maximum BH mass in the initial models, which is regulated by the wind-driven mass loss from the Vink prescription. For the high metallicity models, this produces a large population of $30 M_{\odot} \mathrm{BHs}$, which leads to the formation of a large population of equal-mass mergers with a total mass of $60 M_{\odot}$. More interestingly, we find that massive cluster models produce an additional collection of binaries at $90 M_{\odot}$ and $120 M_{\odot}$, which can be clearly seen at high redshift in Figure 7 . One of the two BHs in these binaries has experienced one and two earlier mergers with lower mass BHs respectively. For the the low metallicity models, there is no apparent collection of sources as might be expected (Rodriguez et al. 2016a). The decreased efficiency of the stellar winds in the low metallicity models implies that a lower number of high mass stars are converted into BHs with the maximum-mass set by the winddriven mass loss prescription, resulting in a wider range of $\mathrm{BH}$ masses.

In Figures 6 and 7, we show the total and chirp mass and uncertainties associated with the recent detection of the BHbinary merger GW150914 (Abbott et al. 2016a). The reported masses of GW150914 are consistent with the masses of black hole mergers from GCs in the local universe. However, even for the low metallicity models, only $5 \%$ of the total number of mergers in GCs produce a merger at low redshift with a total mass significantly larger than $50 M_{\odot}$ as required to match the total mass of the GW150914 event. In NSCs, this percentage is significantly larger, being $\approx 20 \%$ of the total number of inspiraling binaries. In high metallicity clusters (Figure 7) a smaller number of high mass BH mergers is produced at low redshift making these clusters less likely progenitors of GW150914-like events.

In Table 1, we report the mean number of mergers per cluster obtained from our models. NSCs are defined here as clusters with masses in the range $5 \times 10^{6}-5 \times 10^{7} M_{\odot}$, while GCs have masses in the range of $10^{5}-10^{7} M_{\odot}$. In order to obtain the mean rate of mergers, we weighted the number of mergers from each of the cluster models by a cluster initial mass function (CIMF). For GCs, we assume a power law CIMF: $d M / d N \propto M^{-2}$ (e.g., Bik et al. 2003). For NSCs, the initial mass function is largely unknown. Here we take the IMF of NSCs directly from the mass distribution of NSCs at $z=2$ from the galaxy formation models of Antonini et al. (2015b; their Figure 10). These models produce a mass distribution at $z=0$ that is consistent with the observed NSC mass distribution from Georgiev et al. (2016). We note that here we might be underestimating the number of massive mergers from NSCs occurring at low redshift because we have assumed that these systems are as old as Galactic GCs. In fact, while most NSCs appear to be dominated by old stellar components, they are also known to have a complex star formation history and to contain young stellar populations, which can produce high mass mergers also at later times (we will come back to this point below). It is also possible that a large fraction of the NSC stars accumulated gradually in time by infalling GCs that decayed to the center through dynamical friction. If this process is the main mechanism for NSC formation, then NSCs and GCs will comprise similar stellar populations (Antonini 2014).

Table 1 shows that our models predict a few thousand $\mathrm{BH}$ mergers per NSC over $12 \mathrm{Gyr}$ of evolution. This expectation also appears to be consistent with previous estimates (Portegies 

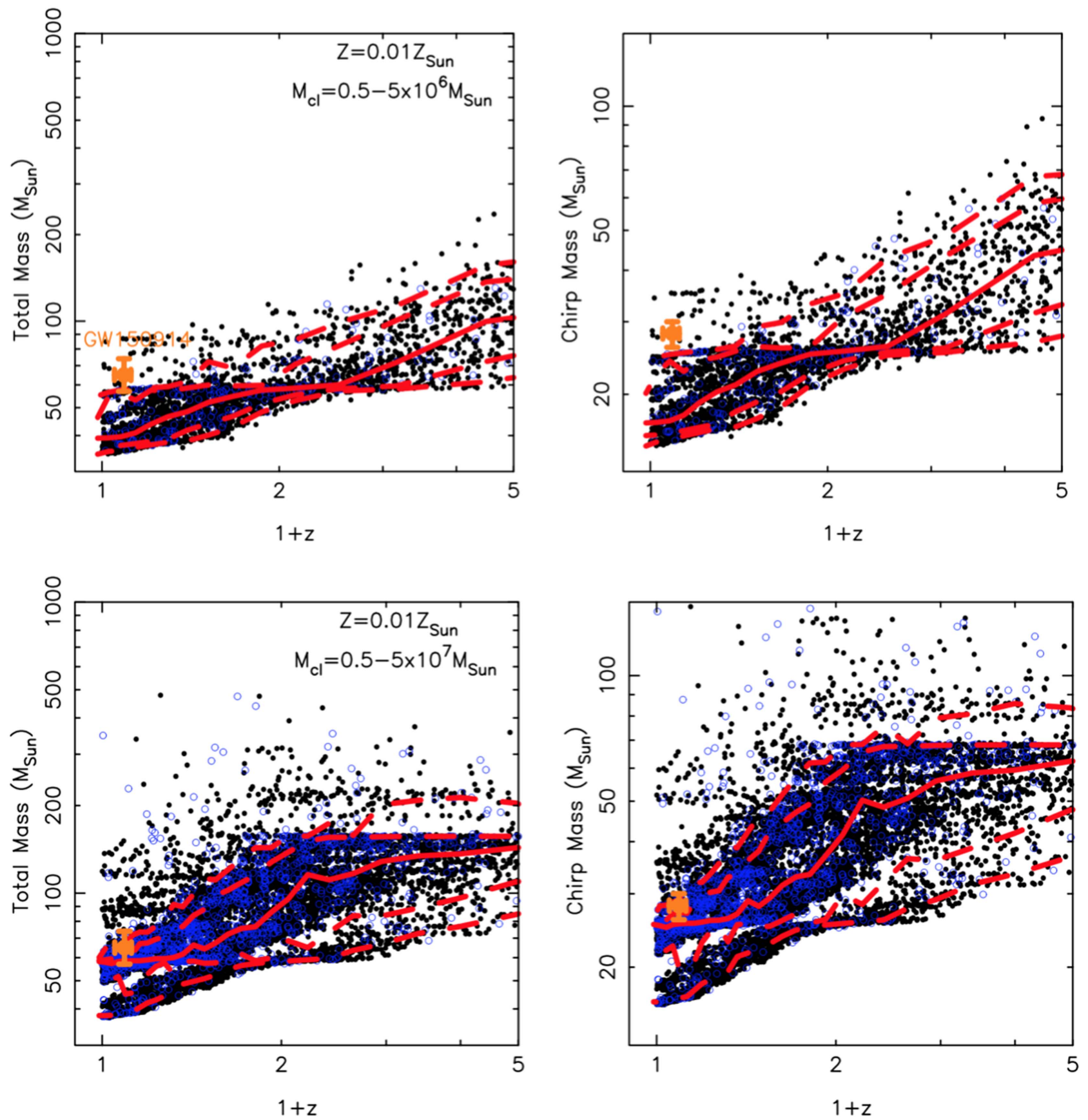

Figure 6. Mass of merging BH binaries for a range of cluster masses that could represent typical GCs (upper panels) or NSCs (lower panels) as a function of redshift. Evolution proceeds from right to left. We assume here that all clusters formed 12 Gyr ago. The uniform spin model described in Section 2.4 was adopted. Open blue circles are systems the are retained inside the cluster after merging. Note how almost all mergers occurring inside low mass clusters are promptly ejected, while for $M_{\mathrm{cl}}=0.5-5 \times 10^{7} M_{\odot}$ many of the inspiraling BHs are expected to be retained inside the cluster.

Zwart \& McMillan 2000; Miller \& Lauburg 2009). In addition, NSCs produce between 50 to $\approx 500 \mathrm{BH}$ mergers with high mass $>50 M_{\odot}$ at $z<0.3$ depending on the $\mathrm{BH}$ spin magnitudes and assumed metallicities distribution of the underlining stellar population. Our GC models produce only a few mergers per cluster within $z<0.3$ and total mass $>50 M_{\odot}$. These massive binaries are found to form only in the most massive GCs $\left(M_{\mathrm{cl}} \gtrsim 10^{6} M_{\odot}\right)$ of low metallicity.

The number of massive mergers at low redshift is also sensitive to the spin magnitude distribution we assume. For high spin models, a smaller number of $\mathrm{BHs}$ are retained in the clusters compared to the uniform spin models. Consequently, high spin models produce fewer high mass $\mathrm{BH}$ mergers at low redshift compared to models that assume low spins. However, in either spin model a number of inspiraling $\mathrm{BH}$ binaries with mass $\gtrsim 50 M_{\odot}$ is found to merge at low redshift. Finally, Table 1 gives the number of $\mathrm{BH}$ mergers that are retained inside the cluster. Between $10 \%$ and $20 \%$ of high mass $\left(>50 M_{\odot}\right)$ mergers occurring in NSCs at $z<1$ are retained inside the cluster enabling the formation of even more massive BH mergers.

The results presented in this section suggest that NSCs are a natural environment for producing $\mathrm{BH}$ mergers that are observable by aLIGO detectors. In addition to this, NSCs can 

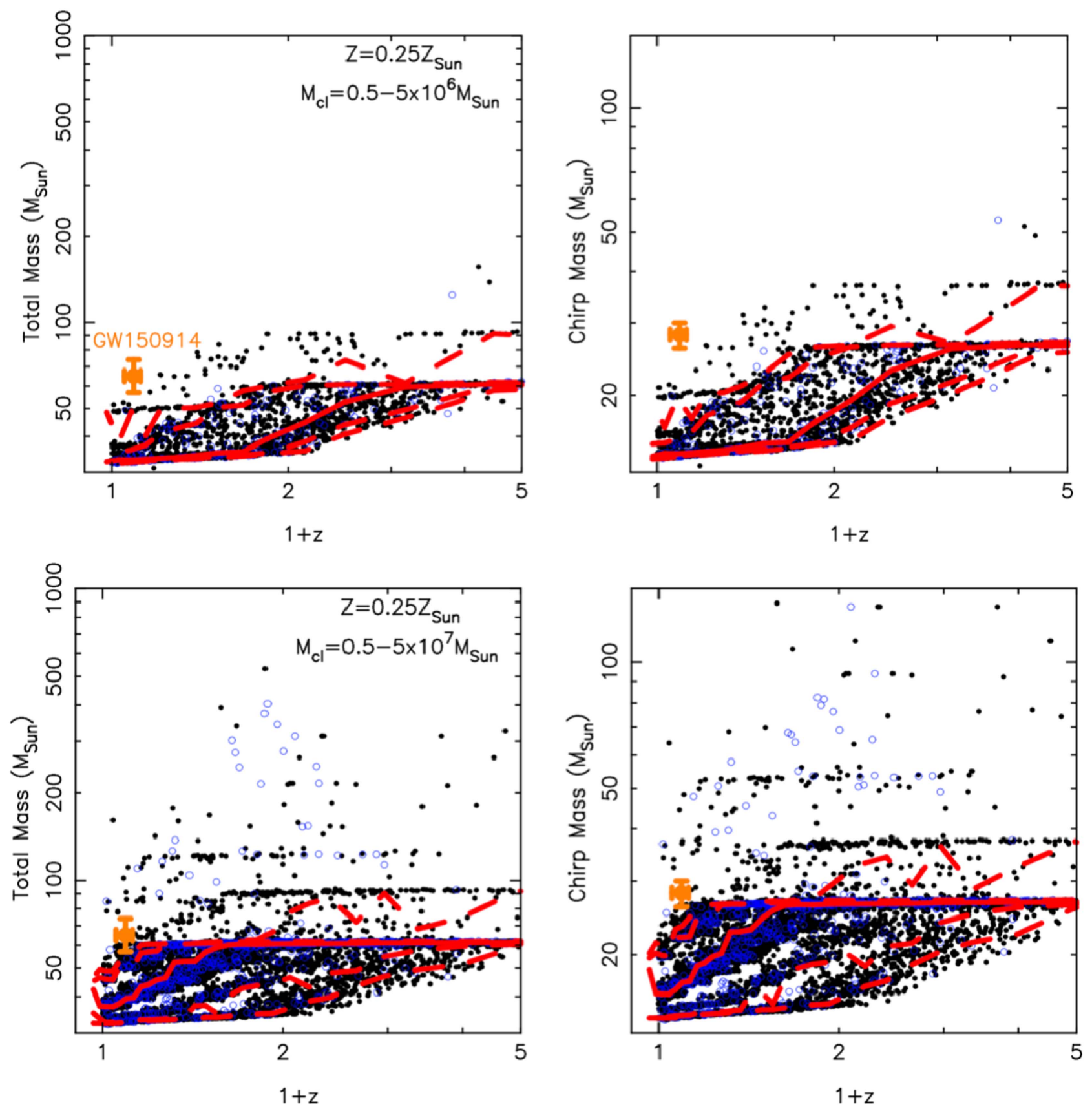

Figure 7. Same as Figure 6 but for $Z=0.25 Z_{\odot}$.

form high mass $\mathrm{BH}$ binaries, and mergers with masses consistent with that of GW150914 also in relatively high metallicity environments. The implications of our results are discussed in more detail in the following section.

\section{IMPLICATIONS AND DISCUSSIONS}

Our study shows that a multitude of BH-binary mergers can be produced at the center of galaxies where NSCs reside. In the following, we derive an approximate expected detection rate from the results of our models and discuss some implications for possible aLIGO detections of these mergers over the next decade. Finally, we discuss the production of $\mathrm{BH}$ mergers in
NSCs hosting a central $\mathrm{MBH}$, and the possibility of a continuous supply of BH binaries in NSCs through episodic and/or continuous star formation.

\subsection{Detection Rate Estimates}

Here we use results from semi-analytical galaxy formation models to derive an expected $\mathrm{MBH}$ occupation fraction in NSCs and use this as well as the results of the cluster semianalytical models presented in this paper to make predictions about the merger rate of $\mathrm{BH}$ binaries produced in NSCs. We also consider the merger rates from GCs and compare these to estimates made in former studies. 
Table 1

The Mean Number of Inspirals per Cluster over 12 Gyr of Evolution, $\langle N\rangle$, That Occur at Redshift $z<0.3$ and $z<1$, and Those That Occur at Redshift $z<0.3$ and $z<1$ and Have a Total Mass $>50 M_{\odot}$.

\begin{tabular}{|c|c|c|c|c|c|}
\hline \multirow[t]{2}{*}{ Model } & \multicolumn{5}{|l|}{$\langle N\rangle$} \\
\hline & $\begin{array}{l}\text { Total } \\
Z=0.01(Z=0.25)\end{array}$ & $\begin{array}{l}z<0.3 \\
Z=0.01(Z=0.25)\end{array}$ & $\begin{array}{l}z<1 \\
Z=0.01(Z=0.25)\end{array}$ & $\begin{array}{l}z<0.3, M>50 M_{\odot} \\
Z=0.01(Z=0.25)\end{array}$ & $\begin{array}{l}z<1, M>50 M_{\odot} \\
Z=0.01(Z=0.25)\end{array}$ \\
\hline NSCs-low spins & 1379 (1307) & $462(450)$ & $990(980)$ & $444(166)$ & $877(506)$ \\
\hline GCs-low spins & $96(80)$ & $23(20)$ & $57(42)$ & $5(0.5)$ & $20(3)$ \\
\hline NSCs_-high spins & $1667(1517)$ & $576(510)$ & $1080(1060)$ & $423(115)$ & $960(490)$ \\
\hline GCs_-high spins & $109(80)$ & $24(20)$ & $55(47)$ & $4(0.2)$ & $20(2)$ \\
\hline \multicolumn{6}{|l|}{ In-cluster Mergers } \\
\hline NSCs-low spins & $0.2(0.2)$ & $0.2(0.2)$ & $0.2(0.2)$ & $0.2(0.2)$ & $0.2(0.2)$ \\
\hline GCs-low spins & $0.07(0.07)$ & $0.07(0.07)$ & $0.07(0.07)$ & $0.04(0.03)$ & $0.07(0.05)$ \\
\hline NSCs_-high spins & $0.07(0.07)$ & $0.07(0.07)$ & $0.07(0.07)$ & $0.1(0.1)$ & $0.1(0.1)$ \\
\hline GCs—high spins & $0.02(0.02)$ & $0.03(0.03)$ & $0.03(0.03)$ & $0.03(0.04)$ & $0.03(0.02)$ \\
\hline
\end{tabular}

Note. Below, we give the fraction of mergers that are retained inside the clusters.

To compute the aLIGO merger rate of BH binaries per unit volume, we use the following expression

$$
\Gamma_{\mathrm{aLIGO}}^{\mathrm{NSC}}=n_{\mathrm{gx}} \Gamma_{\text {merge }} f_{\text {nucleated }}
$$

where $n_{\mathrm{gx}}$ is the number density of galaxies, $\Gamma_{\text {merge }}$ is the averaged merger rate of $\mathrm{BH}$ binaries per cluster that merge within the observable volume, and $f_{\text {nucleated }}$ is the fraction of galaxies, which host an NSC but do not have an MBH.

While observations show that NSCs and MBHs coexist in some galaxies, and that NSCs exist in most galaxies, $f_{\text {nucleated }}$ remains largely unconstrained. Here we use the results of semianalytical galaxy formation models that follow the cosmological evolution of galaxies, their MBHs and NSCs. These galaxy formation models are described in Barausse (2012) and Antonini et al. (2015a, 2015b). Figure 8 shows the fraction of galaxies in these models that contain an NSC but do not host an MBH. These models predict that the number of galaxies hosting an NSC but without an MBH is quite large, being $f_{\text {nucleated }} \gtrsim 0.5$ for galaxies with total mass $M_{\mathrm{Gx}} \lesssim 10^{11} M_{\odot}$ regardless of galaxy type. Based on Figure 8, we adopt here a conservative value of $f_{\text {nucleated }}=0.5$, and adopt a number density of galaxies of $0.02 \mathrm{Mpc}^{-3}$ (e.g., Conselice et al. 2005; Kopparapu et al. 2008). Assuming that BH-BH mergers can be seen by aLIGO out to a redshift of $z \lesssim 0.3$ (Abbott et al. 2016d), which corresponds to an age of the universe of $\approx 10$ Gyr (Planck Collaboration et al. 2015), then Equation (21) gives a merger rate of BHs in NSCs of

$$
\Gamma_{\mathrm{aLIGO}}^{\mathrm{NSC}} \approx 1.5 \mathrm{Gpc}^{-3} \mathrm{yr}^{-1} \text {. }
$$

Thus our calculation predicts a substantial number of detectable BH mergers from NSCs. From Table 1, we see also that between $10 \%$ and $20 \%$ of the total number of merger remnants are retained inside NSCs. Adopting an aLIGO detectionweighted comoving volume of $\approx 10 \mathrm{Gpc}^{-3}$ for full design sensitivity (Abbott et al. 2016d), the rate in Equation (22) translates into a detection rate of $\approx 10 \mathrm{yr}^{-1}$.

The merger rate of Equation (22) can be directly compared to that from GCs:

$$
\Gamma_{\text {aLIGO }}^{\mathrm{GC}} \approx 5 \mathrm{Gpc}^{-3} \mathrm{yr}^{-1},
$$

which was obtained from the number of mergers per GCs within $z<0.3$ in Table 1 and assuming a number density of

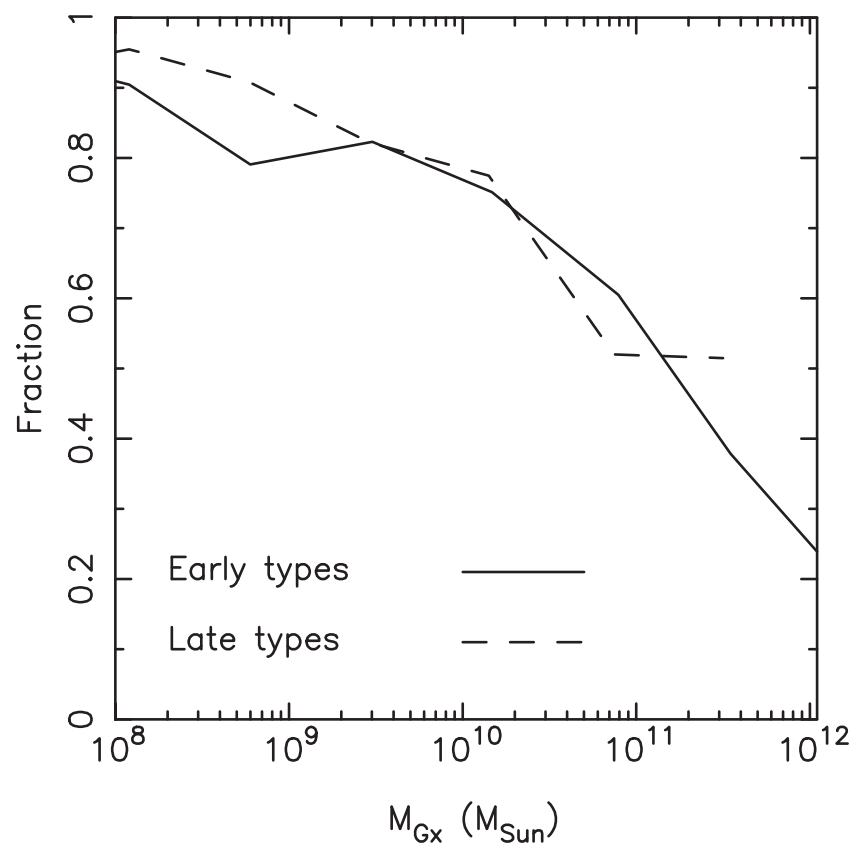

Figure 8. Fraction of galaxies containing an NSC $\left(M_{\mathrm{cl}} \geqslant 10^{5} M_{\odot}\right)$ but no $\mathrm{MBH}$ as a function of galaxy mass. Results are from the galaxy formation models of Antonini et al. (2015a, 2015b). The dashed line is for galaxies with bulge-to-total-mass ratios smaller than 0.7 . The solid line is for early-type galaxies, which are defined here as galaxies with bulge-to-total-mass ratios larger than 0.7 .

GCs equal to $0.77 \mathrm{Gpc}^{-3}$ (Rodriguez et al. 2015). Note that Equation (23) is very well consistent with the rate previously derived by other authors (Rodriguez et al. 2016a). As before, taking an aLIGO detection-weighted comoving volume of $\approx 10 \mathrm{Gpc}^{-3}$ for full design sensitivity, we obtain an aLIGO detection rate of $\mathrm{BH}$ mergers from GCs of $\approx 50 \mathrm{yr}^{-1}$.

Now we only consider mergers with mass $\gtrsim 50 M_{\odot}$ occurring at a redshift $z<0.3$ (see Table 1). We define these as possible progenitors of the event GW150914. The detection rate of high mass mergers from NSCs in the local universe is in the range

$$
\Gamma_{\mathrm{aLIGO}}^{\mathrm{NSC}}\left(z<0.3 ; M>50 M_{\odot}\right) \approx 0.4-1 \mathrm{Gpc}^{-3} \mathrm{yr}^{-1},
$$


where the lower limit corresponds to high metallicity clusters and the high spin model, and the upper limit to low metallicity clusters and to a uniform $\mathrm{BH}$ spin distribution. Interestingly, we find that between $20 \%$ and $50 \%$ of all massive mergers in NSCs are produced by the consecutive merger channel discussed in this paper with a few percent having a mass $\gtrsim 100 M_{\odot}$. The corresponding merger rate of high mass $\mathrm{BH}$ binaries mergers in GCs is

$$
\Gamma_{\mathrm{aLIGO}}^{\mathrm{GC}}\left(z<0.3 ; M>50 M_{\odot}\right) \approx 0.05-1 \mathrm{Gpc}^{-3} \mathrm{yr}^{-1},
$$

similar to that corresponding to high mass $\mathrm{BH}$ mergers produced in NSCs. Both rates of high mass mergers from GCs and NSCs are marginally consistent with the rate of 2-53 $\mathrm{Gpc}^{-3} \mathrm{yr}^{-1}$ of GW150914-like mergers given by Abbott et al. (2016c).

From our rate computation, we find that the detection rate of $\mathrm{BH}-\mathrm{BH}$ binaries from NSCs is substantial, and it is about onetenth of that from GCs. Importantly, we also find that the NSC detection rate of high mass BH mergers similar to GW150914 $\left(M \approx 60 M_{\odot}, z<0.5\right)$ is comparable to that from GCs, with many of the mergers being produced through the consecutive merger scenario discussed in this paper. Finally, our results show that most GW150914-like mergers are more likely to be a product of dynamical interactions occurring in massive clusters of low metallicity, also in agreement with previous findings (Rodriguez et al. 2016b).

\subsection{Dependence on Natal Kicks}

Abbott et al. (2016d) noted that "for both dynamical formation in [globular] clusters and isolated binary evolution, the implication of BH-binary existence is that $\mathrm{BH}$ natal kicks cannot always be high $\left(\gtrsim 100 \mathrm{~km} \mathrm{~s}^{-1}\right)$, in order to avoid disrupting or widening the orbits too much, or ejecting the BHs from clusters before they can interact." For example, large natal kicks will widen the orbits of massive binary progenitors and quench the formation of binary $\mathrm{BH}$ systems that will merge within the age of the universe (e.g., Belczynski et al. 2007). Abbott et al. (2016d) did not consider the possibility that BH binaries can be dynamically assembled inside NSCs, which we discuss here.

Motivated by the fact that the distribution of formation kicks for BHs is largely uncertain, even at the qualitative level, we assume here that the kick magnitude distributions are fully unconstrained and explore how the NSC and GC BH merger rates are affected by varying these distributions. In Figure 9, we plot the mean merger rate per galaxy at $z<1$ in models where the BHs are given natal kicks taken from Maxwellian distributions with dispersion $\sigma_{\text {natal }}$, and the spin magnitude of the BHs is uniformly distributed in the range of $\chi=[0,1)$. Unlike the models discussed above, here we assume that the BH natal kick distributions do not depend on the mass of the BHs.

As $\sigma_{\text {natal }}$ increases above $\gtrsim 20 \mathrm{~km} \mathrm{~s}^{-1}$, the merger rate from GCs decreases and becomes comparable to that of NSCs for $\sigma_{\text {natal }} \approx 50 \mathrm{~km} \mathrm{~s}^{-1}$. Above this value the merger rate of NSCs begins to decrease as well because a fraction of BHs starts to be ejected from low mass NSCs as well. However, for $\sigma_{\text {natal }} \gtrsim 50 \mathrm{~km} \mathrm{~s}^{-1}$, we see that the BH merger rate from NSCs becomes significantly larger than the corresponding merger rate from GCs. We conclude that for high natal kicks, $\gtrsim 50 \mathrm{~km} \mathrm{~s}^{-1}$,

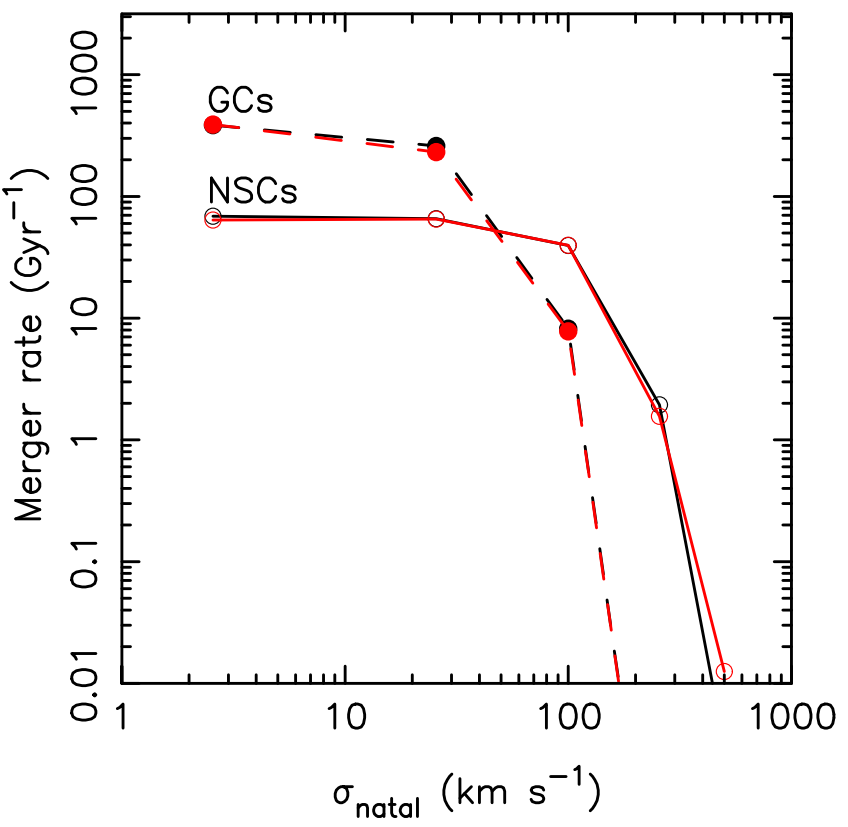

Figure 9. Mean merger rate per galaxy at redshift $z<1$ as a function of the dispersion of the Maxwellian distribution of natal kick velocities, $\sigma_{\text {natal }}$, applied to all BHs independently of mass. Red curves correspond to a metallicity of $Z=0.25 Z_{\odot}$ and black curves to a metallicity of $Z=0.01 Z_{\odot}$. We have assumed a number of GCs per galaxy equal to 100 and a light travel time of $8 \mathrm{Gyr}$ at $z=1$. At $\sigma_{\text {natal }} \gtrsim 50 \mathrm{~km} \mathrm{~s}^{-1}$ the merger rate of $\mathrm{BH}$ binaries from NSCs is dominant compared to the corresponding merger rate from GCs.

NSCs can dominate the merger rate of dynamically formed BH binaries that are detectable by aLIGO.

Recent theoretical studies suggest that the birth kicks may not be directly correlated with the BH mass (Pejcha \& Thompson 2015; Repetto \& Nelemans 2015), and that the distribution of black hole kick velocities could be similar to that of neutron stars $\sigma_{\text {natal }} \approx 200 \mathrm{~km} \mathrm{~s}^{-1}$ (but see Mandel 2016). If this is the case, then the merger rate of BH binaries from NSCs will greatly dominate over that from GCs. However, we also note that for $\sigma_{\text {natal }} \gtrsim 200 \mathrm{~km} \mathrm{~s}^{-1}$ the rate of BH mergers from NSCs becomes significantly smaller than the rate of $2-53 \mathrm{Gpc}^{-3} \mathrm{yr}^{-1}$ implied by the detection of GW150914 (Abbott et al. 2016c). Thus very high values of natal kicks are excluded by our analysis when combined with the estimated aLIGO detection rate.

\subsection{Mergers of Stellar Remnants near MBHs}

So far, we have considered the merger of $\mathrm{BH}$ binaries in stellar clusters that do not host a central MBH. This is certainly not the case for at least a handful of NSCs, which are known to have MBHs at their center (e.g., Seth et al. 2008). One example is our own Galaxy, which contains a $\approx 10^{7} M_{\odot}$ NSC and a central MBH of $\approx 4 \times 10^{6} M_{\odot}$ (Ghez et al. 2008; Gillessen et al. 2009). More generally, NSCs and MBHs are known to coexist in galaxies with masses $\approx 10^{10} M_{\odot}$ (e.g., González Delgado et al. 2008; Seth et al. 2008); galaxies with masses lower than this value show clear evidence for nucleation but little evidence for an MBH. Conversely, galaxies with masses above $10^{11} M_{\odot}$ are dominated by MBHs but generally show no evidence for nucleation (Neumayer \& Walcher 2012).

In NSCs containing a central $\mathrm{MBH}$, the merger rates of $\mathrm{BH}$ binaries are expected to be significantly different, though not necessarily smaller, than the rates given in Table 1 . If an MBH 
is present, it will inhibit core-collapse, causing the formation of a Bahcall-Wolf cusp instead (Bahcall \& Wolf 1976). After the BHs have segregated to the center, their densities will dominate over the stellar densities within a radius $\lesssim 0.1$ times the $\mathrm{MBH}$ influence radius (Hopman \& Alexander 2006; Gualandris \& Merritt 2012) — note that this latter statement depends on the formation history of the NSC (Antonini 2014). At such small distances from the $\mathrm{MBH}$, all binaries will be effectively soft so that any interaction with a third $\mathrm{BH}$ or star will tend to make the binary internal orbit wider. In this situation, three-body interactions between binaries and field objects will not lead to $\mathrm{BH}$ mergers but rather to the "evaporation" of binaries.

Several mechanisms have been discussed in the literature that can produce $\mathrm{BH}$ mergers even in the extreme stellar environment of MBHs. Below, we briefly review two of these processes: (1) mergers of BH binaries due to Lidov-Kozai (LK) resonance induced by the central $\mathrm{MBH}$ (Antonini \& Perets 2012) and (2) single-single captures of compact objects due to GW energy loss (Quinlan \& Shapiro 1990; Lee 1993; O'Leary et al. 2009).

\subsubsection{MBH-mediated BH Mergers in NSCs}

Antonini \& Perets (2012) showed that, near an MBH, the dynamical evolution of binaries is dominated by perturbations from the central MBH. In particular, the LK mechanism (Kozai 1962; Lidov 1962) exchanges the relative inclination of the inner BH-binary orbit to its orbit around the $\mathrm{MBH}$ for the eccentricity of the BH binary (Antonini et al. 2010). At the peak eccentricity, during an LK cycle, GW emission can become efficient, leading to a merger of the two BHs.

More recently, VanLandingham et al. (2016) performed $N$ body simulations of small clusters of stars containing a central $\mathrm{MBH}$, to estimate the rate of $\mathrm{BH}$ mergers, induced by the LK mechanism. These authors found that this mechanism could produce mergers at a maximum rate of $\approx 2$ per megayear per Milky Way equivalent galaxy. This rate appears to be somewhat comparable to the upper end of the expected detection rate from stellar clusters (Rodriguez et al. 2016a). VanLandingham et al. (2016) showed that this rate could translate into a maximum rate per volume of $\approx 100 \mathrm{Gpc}^{-3} \mathrm{yr}^{-1}$. However, as also noted by VanLandingham et al. (2016), their merger rate estimates are likely to be an overestimate of the true merger rate from the LK process because they used optimistic values for both the merger fraction as well as for the BH-binary fraction.

The efficiency of the LK process in inducing BH mergers in NSCs with MBHs is sensitive to major uncertainties. The major challenge to this being that a continuous supply of $\mathrm{BH}$ binaries is needed in order to obtain a finite merger rate. In fact, a continuous formation of binaries is part of the assumptions made in the rate estimates of Antonini \& Perets (2012) and VanLandingham et al. (2016) .

Binaries well inside the influence radius of an $\mathrm{MBH}$ will be essentially all soft and will be disrupted over the typical timescale (Binney \& Tremaine 1987):

$$
\begin{aligned}
t_{\mathrm{ev}}= & \frac{m_{12} \sigma}{16 \sqrt{\pi} G m_{\star} \rho a \ln \Lambda} \approx 10^{7} \frac{\sigma}{100 \mathrm{~km} \mathrm{~s}^{-1}} \\
& \left(0.5 \frac{m_{12}}{m_{\bullet}}\right)\left(\frac{\ln \Lambda}{10} \frac{\rho}{10^{6} M_{\odot} \mathrm{pc}^{-3}} \frac{a}{1 \mathrm{au}}\right)^{-1}
\end{aligned}
$$

Because $t_{\mathrm{ev}}$ is much shorter than the lifetime of any NSC, we expect that most primordial binaries will be disrupted by now in these systems. Antonini \& Perets (2012) describe various processes that may affect the replenishment rate of compact binaries and/or their progenitors in NSCs with an MBH. Perets (2009) suggested that the disruption of triple stars could leave behind a binary in a close orbit around the $\mathrm{MBH}$ and could serve as a continuous source of replenished binaries close to the $\mathrm{MBH}$. In addition, in situ star formation can also repopulate the binary population of NSCs. Central star formation bursts may occur continuously (or episodically) throughout the evolution of the stellar cusp and lead to a steady population of massive binaries near the center. Finally, NSCs might result from the merger of stellar clusters in the inner galactic regions (e.g., Antonini et al. 2015b). Such clusters may harbor an inner core cluster of BHs that formed during the cluster evolution. If these BHs are retained in the cluster, this mechanism may also contribute to the $\mathrm{BH}$ and $\mathrm{BH}$-binary populations in NSCs (Antonini 2014).

\subsubsection{Mergers from $\mathrm{BH}-\mathrm{BH}$ Scattering in NSCs}

O'Leary et al. (2009) showed that in the dense stellar environments, such as those of NSCs $\mathrm{BH}$ binaries, can efficiently form out of $\mathrm{GW}$ radiation during $\mathrm{BH}-\mathrm{BH}$ (singlesingle) encounters. Interestingly, they show that most of the mergers from this channel will have a finite eccentricity while they enter the $10 \mathrm{~Hz}$ frequency band of aLIGO. This processes could become important for sufficiently large cluster masses. However, the predicted rate of $\mathrm{BH}-\mathrm{BH}$ mergers from this channel, though very uncertain, is estimated to be only $\sim 0.01 \mathrm{Gpc}^{-3} \mathrm{yr}^{-1}$ (Tsang 2013), and therefore it is subdominant with respect to the rate from the other processes discussed in this paper. Finally, we note that Antonini et al. (2016) argued that even the rate of eccentric mergers from $\mathrm{BH}-$ $\mathrm{BH}$ scattering in NSCs is likely to be much smaller compared to that of eccentric mergers from $\mathrm{BH}$ triples formed in GCs.

In conclusion, the role of NSCs containing MBHs in producing $\mathrm{BH}$ mergers is a subject of considerable interest, which will likely require high precision $N$-body simulations of a large number of particles. For now, theoretical models suggest that the merger rate of BHs in these systems might be considerably lower than that from NSCs without MBHs.

\subsection{Continuous and Episodic Star Formation in NSCs}

As mentioned above, most NSCs are known to have undergone a complex star formation history characterized by recurrent episodes of star formation (e.g., Walcher et al. 2006). Thus NSCs might still be forming BHs and BH binaries at the present epoch. This is different from GCs, where all stars are old (ages $\gtrsim 10^{10}$ years) and many (if not all) BHs are expected to have been already ejected by now.

As an example, our Galactic center contains a large population of young massive stars, many of which reside in a stellar disk. These stars most likely originated in situ following the fragmentation of a gaseous disk formed from an infalling gaseous clump (e.g., Bonnell \& Rice 2008). Such stars' formation bursts are thought to occur episodically throughout the evolution of the central cusp. Eclipsing and close binaries are observed among the Galactic center young stars, suggesting star formation as an additional process that can repopulate the 
binary population (including BH binaries) in the NSC (Pfuhl et al. 2014).

Observational studies of NSCs in external galaxies, including high resolution spectroscopic surveys, have been used to characterize the star formation history and ages of NSCs (Rossa et al. 2006; Walcher et al. 2006). The common finding emerging from these studies is that most NSCs are characterized by a mixture of morphological components and different stellar populations spanning a wide range of characteristic ages from $10 \mathrm{Myr}$ to $10 \mathrm{Gyr}$. Observations also suggest that the ages of NSCs and masses depend on the host galaxy Hubble type, with NSCs in early-type spirals being older and more massive than those of late-type spirals (Rossa et al. 2006). More generally, the growth of the nuclei might be a continuous and ongoing process occurring during and after most of the host galaxy was formed.

As shown in Figures 6 and 7, most massive BH mergers $\left(\gtrsim 50 M_{\odot}\right.$ ) occur at early times in the lifetime of a single stellar population star cluster. This is because the most massive objects segregate earlier and are ejected earlier through dynamical interactions. This will not be the case if the clusters form new stellar populations at later times. New episodes of star formations in NSCs will lead to the formation of new BHs with a substantial contribution to the merger rate in the local universe. In situ star formation could therefore contribute significantly to the detection rate of the high mass mergers we previously derived (see Equation (24)), though it will likely not affect the total detection rate of $\mathrm{BH}$ mergers in NSCs.

\section{SUMMARY}

Understanding the distribution of $\mathrm{BHs}$ at the centers of galaxies is crucial for making predictions about the expected event rate and source properties for high-frequency $\mathrm{GW}$ detectors. Since the distribution of stellar BHs is not known, and Monte Carlo simulations of star clusters are still limited to a few $10^{6}$ particles, we opted here for a semi-analytical approach, which we used in order to make predictions about the properties and rate of BH-binary mergers that are dynamically assembled in NSCs. In the future, we plan to explore this topic using more accurate, though computationally more demanding, Monte Carlo simulations. The main conclusions of our work are summarized below.

(1) NSCs produce BH-binary mergers at a realistic rate of $\approx 1.5 \mathrm{Gpc}^{-3} \mathrm{yr}^{-1}$.

(2) BHs in NSCs can experience a number of mergers and grow to masses up to a few hundred solar masses. Although rare, such high mass $\mathrm{BH}$ mergers at low redshifts are unique to NSCs, because these are the only clusters with sufficiently high escape velocities such that they can retain a large fraction of their merging BHs.

(3) Assuming that BHs receive low natal kicks, with an imparted momentum equal to the momentum imparted to neutron stars, the NSC detection rate of high mass $\mathrm{BH}$ mergers similar to $\mathrm{GW} 150914\left(M \geqslant 50 M_{\odot}, z \leqslant 0.3\right)$ is $0.4-1 \mathrm{Gpc}^{-3} \mathrm{yr}^{-1}$. This rate is comparable or larger than the corresponding merger rate of dynamically formed $\mathrm{BH}$ binaries in GCs.

(4) If BHs receive natal kicks as large as $\gtrsim 50 \mathrm{~km} \mathrm{~s}^{-1}$, then BH-binary mergers produced dynamically in NSCs could dominate over the merger rate of similar sources produced either in GCs or through isolated binary evolution.

We thank Enrico Barausse, Sourav Chatterjee, Cole Miller, and Carl Rodriguez for useful discussions and the anonymous referee for useful suggestions. F.A. acknowledges support from a CIERA postdoctoral fellowship at Northwestern University. F.A.R. acknowledges support from NSF Grant AST-1312945 and NASA Grant NNX14AP92G, at Northwestern University, and from NSF Grant PHY-1066293 through the Aspen Center for Physics.

\section{REFERENCES}

Aarseth, S. J. 2012, MNRAS, 422, 841

Abbott, B. P., Abbott, R., Abbott, T. D., et al. 2016a, PhRvL, 116, 061102 Abbott, B. P., Abbott, R., Abbott, T. D., et al. 2016b, arXiv:1602.03840 Abbott, B. P., Abbott, R., Abbott, T. D., et al. 2016c, arXiv:1602.03842 Abbott, B. P., Abbott, R., Abbott, T. D., et al. 2016d, ApJL, 818, L22 Antonini, F. 2014, ApJ, 794, 106

Antonini, F., Barausse, E., \& Silk, J. 2015a, ApJL, 806, L8

Antonini, F., Barausse, E., \& Silk, J. 2015b, ApJ, 812, 72

Antonini, F., Chatterjee, S., Rodriguez, C. L., et al. 2016, ApJ, 816, 65

Antonini, F., Faber, J., Gualandris, A., \& Merritt, D. 2010, ApJ, 713, 90 Antonini, F., \& Perets, H. B. 2012, ApJ, 757, 27

Arzoumanian, Z., Chernoff, D. F., \& Cordes, J. M. 2002, ApJ, 568, 289

Bahcall, J. N., \& Wolf, R. A. 1976, ApJ, 209, 214

Banerjee, S., Baumgardt, H., \& Kroupa, P. 2010, MNRAS, 402, 371

Barausse, E. 2012, MNRAS, 423, 2533

Belczynski, K., Dominik, M., Bulik, T., et al. 2010, ApJL, 715, L138

Belczynski, K., Kalogera, V., \& Bulik, T. 2002, ApJ, 572, 407

Belczynski, K., Repetto, S., Holz, D. E., et al. 2016, ApJ, 819, 108

Belczynski, K., Taam, R. E., Kalogera, V., Rasio, F. A., \& Bulik, T. 2007, ApJ, 662, 504

Berti, E., Cardoso, V., Gonzalez, J. A., et al. 2007, PhRvD, 76, 064034

Bik, A., Lamers, H. J. G. L. M., Bastian, N., Panagia, N., \& Romaniello, M. 2003, A\&A, 397, 473

Binney, J., \& Tremaine, S. 1987, Galactic Dynamics (Princeton, NJ: Princeton Univ. Press)

Blandford, R. D., \& Hughes, S. A. 2003, ApJL, 585, L101

Bogdanović, T., Reynolds, C. S., \& Miller, M. C. 2007, ApJL, 661, L147

Böker, T., Sarzi, M., McLaughlin, D. E., et al. 2004, AJ, 127, 105

Bonnell, I. A., \& Rice, W. K. M. 2008, Sci, 321, 1060

Chandrasekhar, S. 1943, ApJ, 97, 255

Chatterjee, S., Fregeau, J. M., Umbreit, S., \& Rasio, F. A. 2010, ApJ, 719,915

Chatterjee, S., Rodriguez, C. L., \& Rasio, F. A. 2016, arXiv:1603.00884

Conselice, C. J., Blackburne, J. A., \& Papovich, C. 2005, ApJ, 620, 564

Côté, P., Piatek, S., Ferrarese, L., et al. 2006, ApJS, 165, 57

de Mink, S. E., \& Mandel, I. 2016, arXiv:1603.02291

Do, T., Kerzendorf, W., Winsor, N., et al. 2015, ApJ, 809, 143

Dominik, M., Belczynski, K., Fryer, C., et al. 2012, ApJ, 759, 52

Dominik, M., Belczynski, K., Fryer, C., et al. 2013, ApJ, 779, 72

Downing, J. M. B., Benacquista, M. J., Giersz, M., \& Spurzem, R. 2011, MNRAS, 416, 133

Duquennoy, A., \& Mayor, M. 1991, A\&A, 248, 485

Figer, D. F., Rich, R. M., Kim, S. S., Morris, M., \& Serabyn, E. 2004, ApJ, 601,319

Fryer, C. L., \& Kalogera, V. 2001, ApJ, 554, 548

Gebhardt, K., Lauer, T. R., Kormendy, J., et al. 2001, AJ, 122, 2469

Georgiev, I. Y., \& Böker, T. 2014, MNRAS, 441, 3570

Georgiev, I. Y., Böker, T., Leigh, N., Lützgendorf, N., \& Neumayer, N. 2016, MNRAS, 457, 2122

Georgiev, I. Y., Hilker, M., Puzia, T. H., Goudfrooij, P., \& Baumgardt, H. 2009, MNRAS, 396, 1075

Ghez, A. M., Salim, S., Weinberg, N. N., et al. 2008, ApJ, 689, 1044

Gillessen, S., Eisenhauer, F., Trippe, S., et al. 2009, ApJ, 692, 1075

González, J. A., Sperhake, U., Brügmann, B., Hannam, M., \& Husa, S. 2007, PhRvL, 98, 091101

González Delgado, R. M., Pérez, E., Cid Fernandes, R., \& Schmitt, H. 2008, AJ, 135, 747

Gualandris, A., \& Merritt, D. 2012, ApJ, 744, 74

Gültekin, K., Miller, M. C., \& Hamilton, D. P. 2004, ApJ, 616, 221

Gültekin, K., Miller, M. C., \& Hamilton, D. P. 2006, ApJ, 640, 156 
Hansen, B. M. S., \& Phinney, E. S. 1997, MNRAS, 291, 569

Harris, W. E. 1996, AJ, 112, 1487

Heggie, D. C. 1975, MNRAS, 173, 729

Hobbs, G., Lorimer, D. R., Lyne, A. G., \& Kramer, M. 2005, MNRAS, 360, 974

Hofmann, F., Barausse, E., \& Rezzolla, L. 2016, arXiv:1605.01938

Hopman, C., \& Alexander, T. 2006, ApJL, 645, L133

Hurley, J. R., Aarseth, S. J., \& Shara, M. M. 2007, ApJ, 665, 707

Hurley, J. R., Tout, C. A., \& Pols, O. R. 2002, MNRAS, 329, 897

Kiel, P. D., \& Hurley, J. R. 2009, MNRAS, 395, 2326

King, I. 1962, AJ, 67, 471

Kopparapu, R. K., Hanna, C., Kalogera, V., et al. 2008, ApJ, 675, 1459

Kozai, Y. 1962, AJ, 67, 591

Lee, M. H. 1993, ApJ, 418, 147

Lee, H. M. 1995, MNRAS, 272, 605

Leigh, N. W. C., Böker, T., Maccarone, T. J., \& Perets, H. B. 2013, MNRAS, 429, 2997

Leigh, N. W. C., Mastrobuono-Battisti, A., Perets, H. B., \& Boumlker, T. 2014, MNRAS, 441, 919

Lidov, M. L. 1962, P\&SS, 9, 719

Lousto, C. O., \& Zlochower, Y. 2008, PhRvD, 77, 044028

Lousto, C. O., Zlochower, Y., Dotti, M., \& Volonteri, M. 2012, PhRvD, 85, 084015

Mandel, I. 2016, MNRAS, 456, 578

Merritt, D. 2009, ApJ, 694, 959

Merritt, D. 2010, ApJ, 718, 739

Merritt, D. 2013, Dynamics and Evolution of Galactic Nuclei (Princeton, NJ: Princeton Univ. Press)

Merritt, D., Ferrarese, L., \& Joseph, C. L. 2001, Sci, 293, 1116

Miller, M. C. 2002, ApJ, 581, 438

Miller, M. C., \& Hamilton, D. P. 2002, MNRAS, 330, 232

Miller, M. C., \& Lauburg, V. M. 2009, ApJ, 692, 917

Miller, M. C., \& Miller, J. M. 2015, PhR, 548, 1
Morscher, M., Pattabiraman, B., Rodriguez, C., Rasio, F. A., \& Umbreit, S. 2015, ApJ, 800, 9

Morscher, M., Umbreit, S., Farr, W. M., \& Rasio, F. A. 2013, ApJL, 763, L15 Neumayer, N., \& Walcher, C. J. 2012, AdAst, 2012, 709038

O’Leary, R. M., Kocsis, B., \& Loeb, A. 2009, MNRAS, 395, 2127

O'Leary, R. M., Meiron, Y., \& Kocsis, B. 2016, arXiv:1602.02809

Pejcha, O., \& Thompson, T. A. 2015, ApJ, 801, 90

Perets, H. B. 2009, ApJ, 698, 1330

Peters, P. C. 1964, PhRv, 136, 1224

Pfuhl, O., Alexander, T., Gillessen, S., et al. 2014, ApJ, 782, 101

Pfuhl, O., Fritz, T. K., Zilka, M., et al. 2011, ApJ, 741, 108

Planck Collaboration, Adam, R., Ade, P. A. R., et al. 2015, arXiv:1502.01582

Portegies Zwart, S. F., \& McMillan, S. L. W. 2000, ApJL, 528, L17

Quinlan, G. D. 1996, NewA, 1, 35

Quinlan, G. D., \& Shapiro, S. L. 1990, ApJ, 356, 483

Repetto, S., Davies, M. B., \& Sigurdsson, S. 2012, MNRAS, 425, 2799

Repetto, S., \& Nelemans, G. 2015, MNRAS, 453, 3341

Rodriguez, C. L., Chatterjee, S., \& Rasio, F. A. 2016a, PhRvD, 93, 084029

Rodriguez, C. L., Haster, C.-J., Chatterjee, S., Kalogera, V., \& Rasio, F. A. 2016b, ApJL, 824, L8

Rodriguez, C. L., Morscher, M., Pattabiraman, B., et al. 2015, PhRvL, 115, 051101

Rossa, J., van der Marel, R. P., Böker, T., et al. 2006, AJ, 132, 1074

Seth, A., Agüeros, M., Lee, D., \& Basu-Zych, A. 2008, ApJ, 678, 116

Sigurdsson, S., \& Hernquist, L. 1993, Natur, 364, 423

Spera, M., Mapelli, M., \& Bressan, A. 2015, MNRAS, 451, 4086

Spitzer, L. 1987, Dynamical evolution of Globular Clusters (Princeton, NJ: Princeton Univ. Press)

Tsang, D. 2013, ApJ, 777, 103

VanLandingham, J. H., Miller, M. C., Hamilton, D. P., \& Richardson, D. C. 2016, arXiv:1604.04948

Walcher, C. J., Böker, T., Charlot, S., et al. 2006, ApJ, 649, 692

Wang, L., Spurzem, R., Aarseth, S., et al. 2015, MNRAS, 450, 4070 